Chapter 20

\title{
Engineering of Inflammation-Resistant Osteochondral Cells
}

\author{
Jan O. Gordeladze, Janne E. Reseland, \\ Tommy A. Karlsen, Rune B. Jakobsen, \\ Astrid K. Stunes, Unni Syversen, Lars Engebretsen, \\ Ståle P. Lyngstadaas and Christian Jorgensen \\ Additional information is available at the end of the chapter \\ http://dx.doi.org/10.5772/55544
}

\section{Introduction}

\subsection{Clinical issue}

Osteoblasts and chondrocytes engineered from various sources of stem cells to replace damaged bone or cartilage in an inflamed joint in patients with inflammatory diseases like osteoarthritis or osteoarthrosis need to be made resilient to the impact of the inflamed environment within the joint cavity (Neve, Corrado et al. 2011; van der Kraan and van den Berg 2012). Clinical experience has clearly shown that the engineered osteochondral cells relatively quickly tend to lose their defined cell phenotype (Chen and Tuan 2008; Kastrinaki and Papadaki 2009), while also tending to perpetuate bone loss through activation of osteoclasts, thus yielding a negative bone remodelling volume (Lories 2011; Goldring 2012). The present concept of manipulation of stem cell microRNA levels envisages one route, by which one may obtain engineered osteochondral cells with a better potential of producing bone and cartilage in vivo, and to withstand the putative detrimental effect of immune cell (e.g. T-helper cell = Th-cell) influence constituted by a plethora of cytokines and exosome-conveyed microRNAs.

\subsection{Previous findings}

We have previously shown that osteochondral cells can be derived from human mesenchymal stem cells (hMSCs) by manipulating the levels of a group of microRNAs, i.e. mir-16, mir-24, mir-125b, mir-149, mir-328, and mir-339 (Gordeladze 2009; Gordeladze, Djouad et al. 2009). All 
these microRNAs are low in differentiated osteoblasts, but highly expressed in stem cells and differentiated chondrocytes. Especially mir-149 seems to serve as a switch, both stimulating chondrogenesis, while suppressing osteoblastogenesis in differentiating hMSCs. Furthermore, mir-328 and mir-339 are the better determinants of the osteochondral cell phenotypes, since manipulations of their intracellular levels enabled us to reciprocally trans-differentiate osteoblasts to chondrocytes at day 7-14 in 2D and 3D cultures in vitro (Gordeladze, Djouad et al. 2009; Gordeladze 2011). Hence, we propose that these six microRNAs, and mir-149, mir-328, and mir-339 in particular, might be able to influence the osteochondral phenotypes in such a way that they are able to maintain their phenotype in vitro and in vivo for an extended period of time, while under the influence of a Th-cell invaded arthritic luminal space.

\subsection{Experimental procedures}

The material used in a defined set of experiments consisted of hMSCs differentiated towards osteoblasts in monolayer cultures or in scaffolds (hydroxyapatite or alginate beads, respectively), hPBMCs (human peripheral blood monocytic cells) differentiated towards osteoclasts and grown on bovine dentine slices, exosomes (100,000xg pellets) from activated Th-17 cells, and SCID mice. The cells were transfected with scrambled microRNA, mir-16, mir-24, mir-125b, mir-149, mir-328 or mir-339 pre-microRNAs or antago-microRNAs, using amounts known to suppress or enhance endogenous levels approximately 5 times (Gordeladze 2009). Human MSCs, osteoblasts or chondrocytes were grown in a medium supplemented with chemokines (Interleukin-1 = IL-1, Interleukin-6 = IL-6, and Interleukin-17A = IL-17A, and $\mathrm{TNF} \alpha$ ), or over-layered with exosomes from Th17 cells, either in the absence or presence of dentine slices over-layered with osteoclasts differentiated from hPBMCs, using RANK-L and M-CSF (Fritz, Brondello et al. 2011). Subsequent to a certain period of time (7-21 days), osteochondral cells were either analysed for phenotypic characteristics (e.g. matrix production, microRNA levels, phenotypic gene expression) or injected (without or with mature osteoclasts) into the tibial muscle of SCID mice. After 14 days of in vivo incubation, the de novo formed osteochondral tissues were analysed for phenotypic markers of injected cells by Q-PCR, as well as matrix characteristics (e.g. matrix protein immunohistochemistry, hydroxyapatite production, or GAG = glucosaminglycan over DNA ratio).

\subsection{Results}

These experiments showed the following:

1. Human MSCs, pre-chondrocytes or pre-osteoblasts lost many of their phenotypic characteristics as matrix builders upon exposure to either pre-microRNAs (osteoblasts) or antago-microRNAs (chondrocytes).

2. These cells, when grown in co-culture with differentiated osteoclasts, appeared to enhance osteoclast multi-nucleation, gene expression, and ensuing dentine resorption surface area.

3. By exposing these cells to either artificial, i.e. "inflamed" synovial fluid (growth medium fortified with Th17 cell like profile of cytokines), exosome-like fractions obtained from activated Th17 cells, or certain pre-microRNAs (i.e. mir-222 for chondrocytes or mir-22 
for osteoblasts), we observed that the osteochondral phenotypes were disturbed, along with a concomitantly enhanced potential for osteoclast activation.

4. Growing hMSCs in a 3D structure (hydroxyapatite for osteoblasts and soft alginate beads for chondrocytes) also stabilized phenotypic characteristics, though to a lesser degree.

5. Injecting manipulated osteochondral cells with or without a certain number of predifferentiated osteoclasts into the tibial muscle of SCID mice yielded de novo formed bone and cartilage tissues, where more than $95 \%$ of the injected cells resided within the newly formed tissues. Parameter evaluation (histology, immuno-histochemistry of collagens and aggrecan, X-ray analysis, GAG/DNA ratio, estimation of total $\mathrm{Ca}^{2+}$-contents, and QPCR analyses of marker genes) of the osteochondral cells, as well as Q-PCR analyses of osteoclast marker genes (like the calcitonin receptor, TRAP, cathepsin K, and carbonic anhydrase II) clearly showed that either of the de novo formed tissues were not functioning optimally, and that enhanced osteoclast activation prevailed.

6. Interestingly, cells having been transfected with optimal amounts of either pre-microRNAs or antago-microRNAs maintained their phenotypic characteristics within the tibial muscle of SCID mice, even when pre-exposed to the "inflammation"-mimicking medium or exosome-like particles from activated Th17 cells.

7. Polycistronic vector constructs containing the pre- and antago-microRNAs 149, 328, and 339 are now being made to verify the benefit of controlling the endogenous levels of these microRNA species to produce stable osteochondral phenotypes to be implemented in in vitro and in vivo animal model systems.

\subsection{Conclusion}

Manipulating pertinent microRNAs in osteoblast or chondrocytes derived from hMSCs (or other sources, such as hADSCs = human adipose tissue derived stem cells) may facilitate differentiation to proper, functional cell phenotypes, which are also resilient to the detrimental impact from inflammatory chemokines and/or microRNAs, proteins or mRNAs transferred from Th-cells (via exosome-like vehicles) to engineered osteoblasts or chondrocytes.

\section{Bone remodelling}

\subsection{Cellular and molecular mechanisms of bone remodelling}

Physiological remodelling of bone has proven to be a highly coordinated process constituted by bone resorption following bone formation, and it is necessary for damaged bone to be repaired, and to maintain mineral homeostasis (Mackie, Ahmed et al. 2008; Kular, Tickner et al. 2012; Thompson, Rubin et al. 2012). In addition to the traditional cells localized within the bone tissue (i.e. osteoclasts, osteoblasts, and osteocytes) necessary for completion of multiple bone remodelling cycles, several classes of immune cells have also been implicated in bone turnover and also bone disease states (Chen and Tuan 2008; Lories 2011; Mellis, Itzstein et al. 
2011; Sims and Walsh 2012). The two processes of modelling and remodelling ensure proper development and maintenance of the skeletal system. The process of bone modelling is mandatory for growth and mechanically induced adaption of bone (Kular, Tickner et al. 2012; Thompson, Rubin et al. 2012), but it requires that the processes of bone formation and bone resorption (removal of "old" bone with micro-fractures) occur independently, and at distinct locations within the skeleton (Chang, Raggatt et al. 2008; Raggatt and Partridge 2010; Kular, Tickner et al. 2012). These tightly coordinated events require the synchronized activities of multiple cellular participants and compartments, in order to ensure that bone resorption and formation occur sequentially within the same anatomical locations to preserve bone mass with a functional geometry.

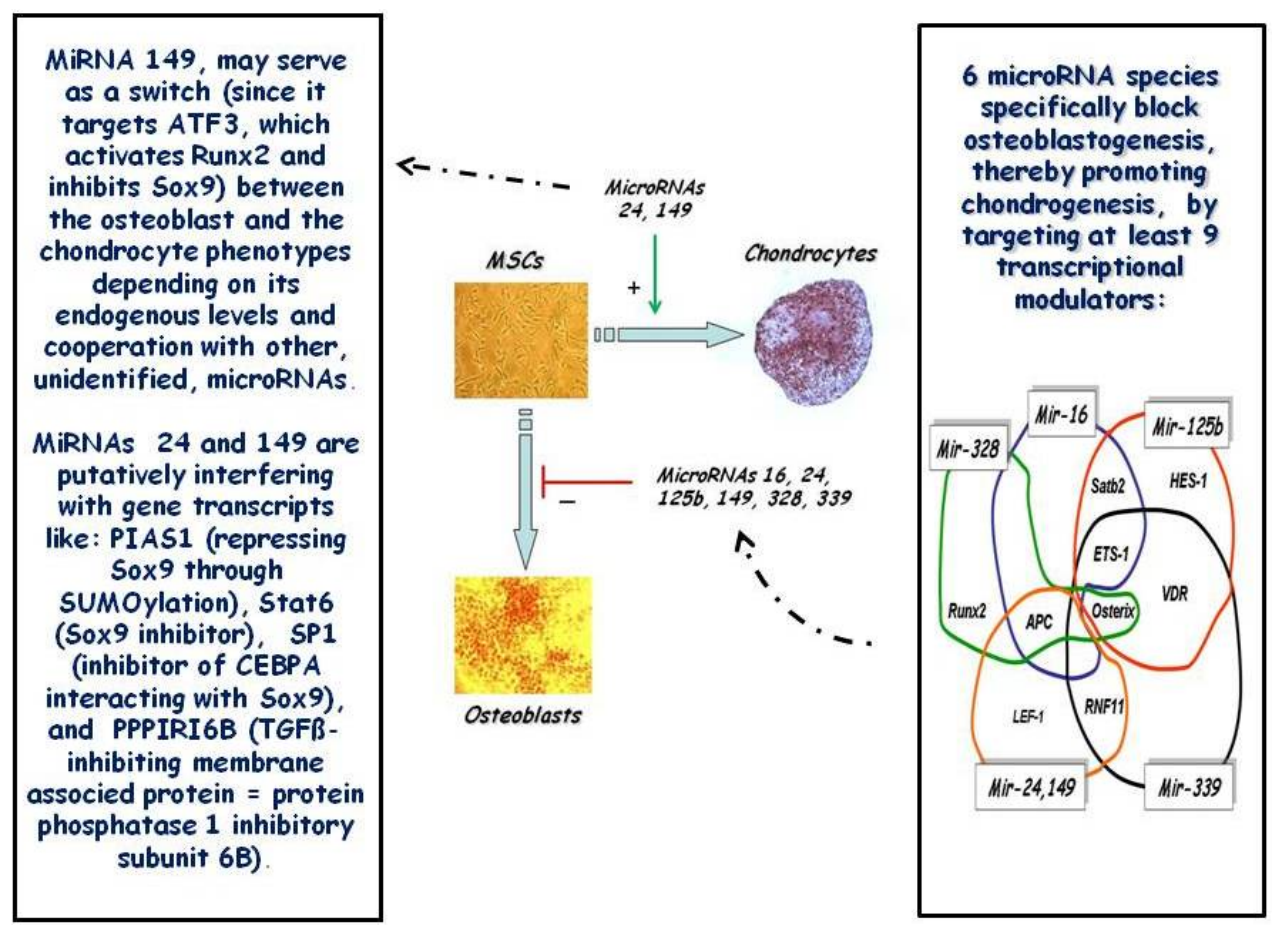

Figure 1. Schematic representation of a previously published microRNA profile distinguishing between osteoblasts and chondrocytes differentiated from human mesenchymal stem cells (hMSCs). Right hand frame: The six microRNA species have been proven to bind to and suppress the transcription of genes encoding nine factors deemed important for osteoblastogenesis, i.e. SATB2, HES-1, ETS-1, RUNX2, APC2, OSTERIX, VDR, LEF-1, and RNF11 (Marie 2008; Gordeladze, Djouad et al. 2009). Left hand frame: Interestingly, the microRNA species mir-24, and especially mir-149, may be regarded as important "switches" between the osteoblast and chondrocyte phenotypes, since they both positively suppress transcription factors (TFs), as predicted by using the MirnaViewer and the Sanger algorithms, necessary for osteoblastogenesis, and at the same time negatively affect factors (like ATF4, PIAS1, STAT6, and PPPIR6B) (Legendre, Dudhia et al. 2003; Komatsu, Mizusaki et al. 2004; Oh, Kido et al. 2007; Suzuki, Osumi et al. 2010), which serve as inhibitors of Sox9 expression, deemed as the most important TF initiating chondrocytogenesis and maintaining the chondrocyte phenotype. 


\subsection{Cells being involved in bone remodelling}

Osteoblasts - Osteoblasts are specialized bone-forming cells responsible for many of the crucial functions constituting bone remodelling (i.e. expression of osteoclastogenic factors, production of bone matrix proteins, and bone mineralization) (Karsenty 2008). The stage of differentiation acquired by the osteoblasts influences the functional contribution to and impact of these cells on in vivo bone remodelling. Immature pre-osteoblasts influence osteoclastogenesis, while mature osteoblasts are responsible for matrix production and mineralization. Osteoblasts develop from pluripotent hMSCs, which exhibit inherent potential to develop into adipocytes, myocytes, chondrocytes, and osteoblasts orchestrated by a series of defined transcription factors (TFs). Osteoblast differentiation has since long been reported to be controlled by RUNX2, but several other TFs are now recognized as equally important for osteoblastogenesis (Franceschi, Xiao et al. 2003). It has been published that 14 different TFs (and/or modulatory proteins), including SATB2, HES-1, ETS-1, LEF-1, SP7 (OSTERIX), RNF11, VDR, and APC2 (a positive regulator of $\beta$-catenin of the canonical WNT-pathway) define the differentiating potential of osteoblasts with a statistical extremely high probability $\left(\mathrm{p}=2 \alpha<5 \times 10^{-13}\right.$ ) (Gordeladze, Djouad et al. 2009).
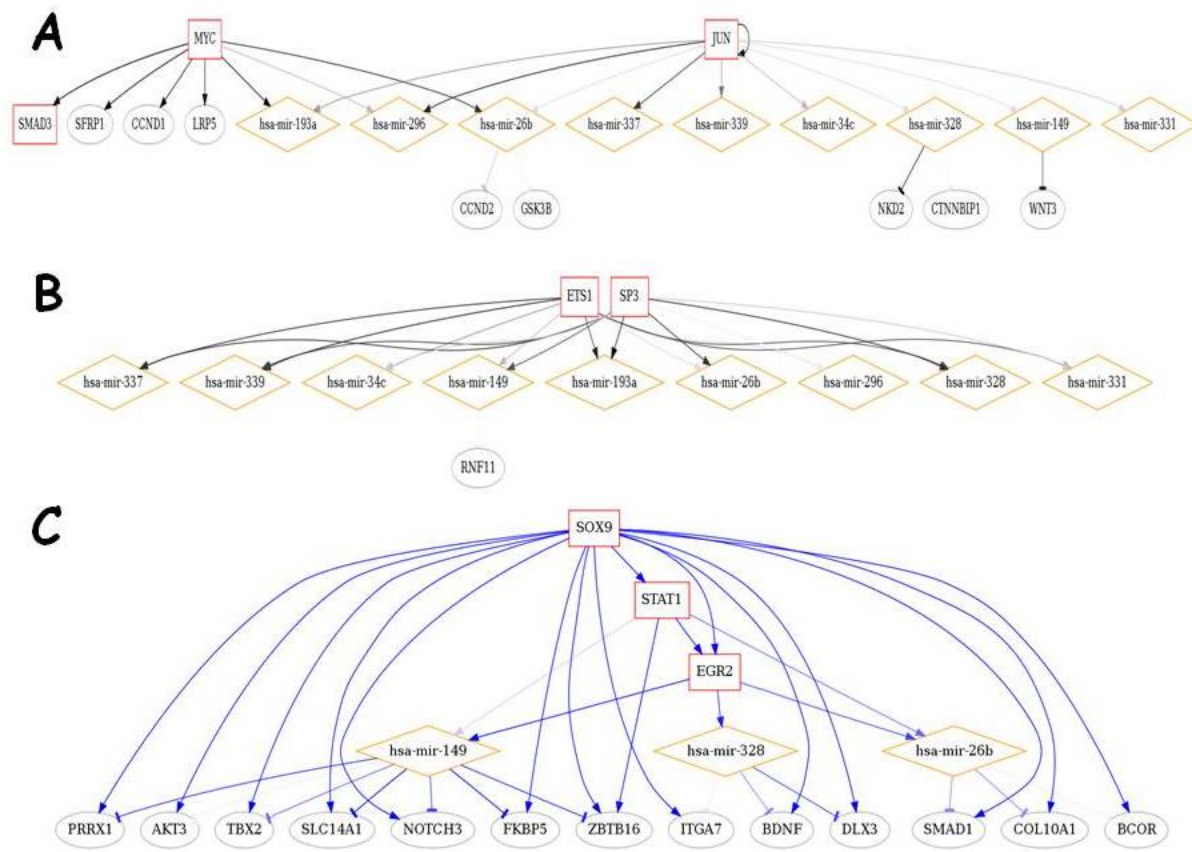

Figure 2. Putative interactions between transcription factors (TFs), microRNA species, and functional genes describing: (A). Hierarchical feed-forward regulation by MYC (Myc proto-oncogene protein) and JUN (transcription factor AP-1) of the canonical WNT-system (important for osteoblastogenesis), including factors like SMAD3 (modulator of TCF/LEF), SFRP1 (secreted frizzled-related protein 1), CCND1 (Cyclin D1), LRP5 (low density lipoprotein receptor-related protein 5), hsa-mir-339, hsa-mir-328, and hsa-mir-149 directly, while CCND2 (cyclin D2), GSK3B (glycogen syn- 
thase kinase 3ß), NKD2 (naked cuticle 2), CTNNBIP1 (catenin $\beta 1$ ), and WNT3 (wingless-type MMTV integration site family, member 3) are affected indirectly. (B). Hierarchical feed-forward regulation by ETS1 (a stimulator of CCN2 = connective tissue growth factor, a mediator of TGF 31 -mediated matrix protein synthesis in osteoblasts) and SP3 by microRNA species including hsa-mir-339, hsa-mir-149, and hsa-mir-328. The TF RNF11, which has now received increasing attention as a major modulator of osteoblastogenesis (Gao, Ganss et al. 2005), is directly affected by hsamir-149. (C). Hierarchical (and possibly superior) feed-forward regulation by Sox9 of STAT1- and EGR2-mediated transcription of the micro-RNA species mir-149 and mir-328. EGR2/KROX-2 (a transcription factor stimulated by WNT3) exerts a major impact on mir-149 and mir-328, which directly affect the transcription of genes like PRRX1, AKT3, TBX2, SLC14A1, NOTCH3, FKBP5, BDNF, and DLX3 (as predicted using the MirnaViewer and Sanger algorithms. Of these genes, PRRX1 is a regulator of OSTERIX, AKT3 and RUNX2 (Lu, Beck et al. 2011) where all are mutually involved in osteoblast differentiation, TBX2 is required for osteoblast proliferation, NOTCH3 inhibits osteoblast differentiation(Lin and Hankenson 2011), FKBP5 overexpression is known to ensure osteoblast differentiation from hMSCs, BDNF is involved in endochondral ossification (Yamashiro, Fukunaga et al. 2001; Marie 2008), and DLX3 is well known as an early inducer of osteoblast differentiation co-acting with RUNX2, DLX5, and MSX2 (Heining, Bhushan et al. 2011). This particular scheme of interactions emerged when combining an osteoblast microRNA micro-array with published osteoblast mRNA transcriptomes (Gordeladze 2011). The bioinformatics modulations are performed by using the algorithm Mir@nt@n (Le Bechec, Portales-Casamar et al. 2011) with standard stringent conditions for putative modulations of gene expression. As the reader will notice, a few other microRNA species may also have been selected to be included amongst the six microRNAs constituting the present osteochondral signature.

Osteocytes - During the course of bone formation, a sub-population of osteoblastic cells undergoes terminal differentiation and becomes engulfed within unmineralized osteoid. They are referred to as osteocytes (Palumbo 1986). Following the mineralization phase, these entombed cells form a network which extends throughout the mineralized bone tissue. Osteocytes respond to mechanical load (mechano-stimulation), and their network is believed to integrate the detection bone micro-damage (micro-fractures within the mineralized bone), which accumulates as a result of normal skeletal loading and fatigue (Verborgt, Tatton et al. 2002). Data have been obtained that support the idea that osteocytes initiate and direct the subsequent remodelling process that repairs damaged bone. Furthermore, the osteocytes have been hypothesized to serve as sensors for the nervous system connected to the skeleton, and for instance convey hypothalamus-derived nerve impulses driven by leptin receptors as a feedback regulatory loop controlling bone mass (i.e. bone turnover) within optimal limits (Takarada and Yoneda 2008; Thompson, Rubin et al. 2012; Motyl and Rosen 2012).

T-cells and B-cells - T-lymphocytes and B-lymphocytes serve as central players of the adaptive immune system facilitating the recognition and the destruction of pathogenic microorganisms. Mice, which are devoid of either B- or T-lymphocytes exhibit osteoporosis, indicating that these immune cells are routinely participating in the maintenance of bone homeostasis ( $\mathrm{Li}$, Toraldo et al. 2007). Mature B-cells produce $~ 50 \%$ of total bone marrow-derived OPG, which would contribute significantly to the restraint of osteoclast differentiation and activation during normal bone turnover. However, the role of T-cells in regulating bone remodelling during homeostasis is less clear. Based on data that T-cell-deficient CD40 knock-out and CD40L knockout mice are osteoporotic ( $\mathrm{Li}$, Toraldo et al. 2007), it has been proposed that T-cells work cooperatively with B-cells and enhance OPG production via CD40/CD40L co-stimulation (Raggatt and Partridge 2010)

Osteomacs - Osteomacs are defined as tissue macrophages residing on on within the endosteal and/or periosteal surfaces. In vitro, osteomacs are required to ensure full functional differentiation of the osteoblast, i.e. the acquisition of mineralizing properties, which consti- 
tutes the late phase of the osteoblasts' life span. In vivo, osteomacs form a canopy over mature matrix-producing osteoblasts at sites of bone modelling, which serves as an ideal anatomical location from which to regulate such a process. A depletion of macrophages in vivo inevitably leads to a complete loss of endosteal osteomacs and associated osteoblasts, indicating that the osteomacs are needed to sustain osteoblast maturation and mineralizing property (Chang, Raggatt et al. 2008).

Osteoclasts - Osteoclasts are terminally differentiated myeloid derived cells, being adapted to excavate mineralized osteoid (bone matrix). These particular cells exhibit distinct morphological and phenotypic characteristics routinely used as functional markers. They include multinuclearity and expression of tartrate-resistant acid phosphatase (TRAP) and the calcitonin (CT) receptor (Teitelbaum and Ross 2003). M-CSF (macrophage colony-stimulating factor) and RANK-L (receptor activator of NFK-B ligand) serve as critical cytokines being required for the survival, expansion, and the differentiation of osteoclast precursors (hPBMCs = human peripheral blood monocytic cells) in vitro (Lacey, Timms et al. 1998). The requirement of these cytokines for osteoclast induced bone resorption in vivo, has been demonstrated in animal model systems lacking functional M-CSF and RANK-L. The molecule OPG-2 (osteoprotegerin-2) serves as a soluble decoy receptor for RANK-L and a negative physiological regulator of osteoclastogenesis, i.e. loss of functional OPG in mice, results in osteoporosis, due to excessive induction (or differentiation) and activation of osteoclasts (Simonet, Lacey et al. 1997). The current paradigm asserts that the RANK-L/OPG expression ratio determines the degree of the osteoclasts' biological activity (i.e. their differentiation and function) (Hofbauer, Khosla et al. 2000). Several TFs, like FOS, MYC, AP-1 (JUN), PU.1, and CREB (Tondravi, McKercher et al. 1997) are deemed necessary to differentiate myeloid cells toward the osteoclast phenotype. MITF (microphthalmia-associated transcription factor) and NFATc1 (nuclear factor of activated T-cells, cytoplasmic 1), which is also important for hMSCs to develop into the osteoblastic lineage (Marie 2008) are also required for osteoclast formation and their expression of osteoclast-specific genes like TRAP (Luchin, Purdom et al. 2000), cathepsin K, and the CT receptor (Matsumoto, Kogawa et al. 2004; Hu, Sharma et al. 2007).

\subsection{The process of bone remodelling}

Prior to activation, the resting bone surface is covered with bone-lining cells, including preosteoblasts intercalated with osteomacs. B-cells are present in the bone marrow and secrete OPG, which suppresses osteoclastogenesis (Raggatt and Partridge 2010).

Activation: The endocrine bone-remodelling signal PTH binds to the PTH receptor on preosteoblasts. Damage to the mineralized bone matrix results in localized osteocyte apoptosis, reducing the local TGF- $\beta$ concentration and its inhibition of osteoclastogenesis (Juppner, Abou-Samra et al. 1991; Heino, Hentunen et al. 2002; Swarthout, D'Alonzo et al. 2002; Verborgt, Tatton et al. 2002; Aguirre, Plotkin et al. 2006; Bonewald 2007; Weitzmann and Pacifici 2007).

Resorption: In response to PTH signalling, monocyte chemotactic protein (MCP-1) is released from osteoblasts and recruits pre-osteoclasts to the bone surface. Additionally, osteoblast expression of OPG is diminished, and production of M-CSF and RANK-L is increased, in order to promote proliferation of osteoclast precursors (hPBMCs) and differentiation of mature 


\begin{tabular}{|c|c|c|c|c|c|c|}
\hline MiteroRNA species & 16 & 24 & $125 b$ & 149 & 328 & 339 \\
\hline $\begin{array}{l}\text { Fold miRNA alteration } \\
\text { in chondrocytes using } \\
\text { PremiRNAs }\end{array}$ & $6 \pm 2$ & $8 \pm 3$ & $6 \pm 3$ & $9 \pm 3$ & $7 \pm 1$ & $8 \pm 2$ \\
\hline $\begin{array}{l}\text { Effect on: Sox9, } \\
\text { Coll2a1, Gli3, } \\
\text { GAG/DNA }\end{array}$ & $132 \pm 17$ & $91 \pm 13$ & $108 \pm 8$ & $120 \pm 18$ & $110 \pm 8$ & $129 \pm 16$ \\
\hline $\begin{array}{l}\text { Fold miRNA alteration } \\
\text { in chondroeytes using } \\
\text { AntagomiRNAs }\end{array}$ & $0.12 \pm 0.03$ & $0.14 \pm 0.03$ & $0.13 \pm 0.06$ & $0.12 \pm 0.05$ & $0.14 \pm 0.06$ & $0.11 \pm 0.04$ \\
\hline $\begin{array}{l}\text { Effect on: Sox9, } \\
\text { Coll2a1, Gli3, } \\
\text { GAG/DNA }\end{array}$ & $18 \pm 5$ & $278 \pm 35$ & $17 \pm 7$ & $191 \pm 31$ & $16 \pm 5$ & $19 \pm 4$ \\
\hline $\begin{array}{l}\text { Fold miRNA alteration } \\
\text { in osteoblasts using } \\
\text { PremiRNAs }\end{array}$ & $9 \pm 4$ & $8 \pm 3$ & $8 \pm 3$ & $7 \pm 2$ & $9 \pm 2$ & $7 \pm 3$ \\
\hline $\begin{array}{l}\text { Effect (relative to } \\
\text { control) on: Run } \times 2 \text {, } \\
\text { Coll1a1: OC \% Min Surf }\end{array}$ & $13 \pm 6$ & $17 \pm 5$ & $14 \pm 3$ & $15 \pm 7$ & $15 \pm 6$ & $12 \pm 4$ \\
\hline $\begin{array}{l}\text { Fold miRNA alteration } \\
\text { in osteoblasts using } \\
\text { AntagomiRNAs }\end{array}$ & $0.40 \pm 0.13$ & $0.38 \pm 0.16$ & $0.41 \pm 0.14$ & $0.49 \pm 0.12$ & $0.43 \pm 0.14$ & $0.53 \pm 0.17$ \\
\hline $\begin{array}{l}\text { Effect (relative to } \\
\text { control) on: Run } \times 2 \text {, } \\
\text { Coll1a1: OC } \% \text { Min Surf }\end{array}$ & $237 \pm 42$ & $192 \pm 31$ & $227 \pm 22$ & $210 \pm 26$ & $198 \pm 22$ & $231 \pm 32$ \\
\hline
\end{tabular}

Figure 3. The impact of pre-microRNA and antago-microRNA species of the osteochondral microRNA signature on the differentiation of osteochondral cells from hMSCs. The following parameters were analysed: endogenous microRNA levels, and average alteration in various differentiation markers. MicroRNA levels and marker gene mRNAs (Collagens, Gli3, Sox9, Osteocalcin, and Runx2) were analysed using the Q-PCR method (Ambion). Estimations of GAG/DNA-ratio (chondrocytes only) were performed using colorimetric methods. Mineralized surface calculations in osteoblast cultures were achieved using the Alizarin red S staining method (Gordeladze, Noel et al. 2008)

osteoclasts. The mature osteoclastic cells become anchored to RGD-binding sites (Arg-Gly-Asp containing proteins), creating a localized micro-environment (i.e. a sealed zone) facilitating the degradation of mineralized bone matrix (Partridge, Jeffrey et al. 1987; Insogna, Sahni et al. 1997; Saftig, Hunziker et al. 1998; Burgess, Qian et al. 1999; McHugh, Hodivala-Dilke et al. 2000; Teitelbaum 2000; Ma, Cain et al. 2001; Yang, Chien et al. 2004; Li, Qin et al. 2007).

Reversal: So called reversal cells engulf and destroy demineralised, undigested collagen from the bone surface. Transition signals are generated for the purpose of halting bone resorption and to stimulate the process of bone formation(Tran Van, Vignery et al. 1982; Kraal, Rep et al. 1987; Heinemann, Siggelkow et al. 2000; Everts, Delaisse et al. 2002; Takahashi, Takahashi et al. 2004; Newby 2008).

Formation: Signalling molecules stimulating bone formation arise from degraded bone matrix, mature osteoclastic cells, and potentially also reversal cells. PTH-binding to and mechanostimulation of osteocytes reduce the expression and secretion of sclerostin (an inhibitor of BMP-binding to its receptor), allowing Wnt-stimulated bone formation to occur (Fermor and Skerry 1995; Harmey, Hessle et al. 2004; van Bezooijen, Roelen et al. 2004; Li, Zhang et al. 2005; Martin and Sims 2005; Murshed, Harmey et al. 2005; Zhao, Irie et al. 2006; Pederson, Ruan et al. 2008; Robling, Niziolek et al. 2008; Tang, Wu et al. 2009). 


\section{A}
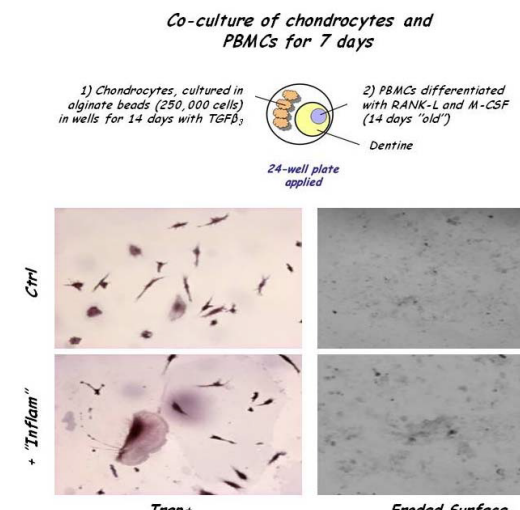

4-well plate
applied
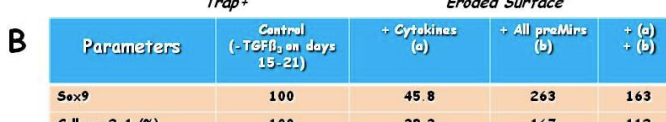

\begin{tabular}{|l|l|}
\hline Collagen2a1 (\%) & 1 \\
\hline Aggrecan & 1 \\
\hline Runx2 & 1 \\
\hline Rank-L & 100 \\
\hline
\end{tabular}

Rank-L

OPG

Clinical score

Trap+ cells $\geq 3$ nucl.

Eroded surface

Mir-16

Mir-24

Mir-125b

Mir-149

Mir- 328

Mir-339

38. 3

45.6

234

287

100

100
100

$300-34.0$

3.2

3.2
22.1

12.1

12.1
7.3

7.3
100

100

100

100

100

100

100

\begin{tabular}{|c|c|}
\hline 167 & 112 \\
\hline 188 & 134 \\
\hline 112 & 132 \\
\hline 156 & 106 \\
\hline 7.9 & 133 \\
\hline 8.2 & 6.5 \\
\hline 5.8 & 13.2 \\
\hline 457 & 9.4 \\
\hline 488 & 145 \\
\hline 512 & 178 \\
\hline 445 & 154 \\
\hline 565 & 298 \\
\hline 512 & 88.9 \\
\hline
\end{tabular}

C

\begin{tabular}{|c|c|c|c|c|}
\hline Parameters & $\begin{array}{l}\text { Control } \\
(-3 M P 22 \text { on days } \\
15-21)\end{array}$ & $\begin{array}{l}+ \text { Cytakines } \\
\text { (o) }\end{array}$ & + All antogomirs & $\begin{array}{l}+(0) \\
+(b)\end{array}$ \\
\hline
\end{tabular}

\begin{tabular}{|l|c|c|c|c|}
\hline Runx2 & 100 & 55.7 & 251 & 147 \\
\hline Collagen1a1 & 100 & 28.5 & 155 & 124 \\
\hline Osteocalcin & 100 & 35.9 & 173 & 145 \\
\hline Osterix & 100 & 64 & 182 & 132 \\
\hline Rank-L & 100 & 287 & 112 & 111 \\
\hline OPG & 100 & 53.3 & 176 & 121 \\
\hline Mineralizing surface & 67.0 & 23.1 & 95.3 & 72.7 \\
\hline Trap+ cells 23 nucl. & 13.3 & 27.3 & 14.8 & 15.1 \\
\hline Eroded surface & 12.6 & 28.7 & 10.9 & 11.7 \\
\hline Mir-16 & 100 & 112 & 21.1 & 112 \\
\hline Mir-24 & 100 & 147 & 31.3 & 118 \\
\hline Mir-125b & 100 & 118 & 21.7 & 114 \\
\hline Mir-149 & 100 & 112 & 18.5 & 92.2 \\
\hline Mir-328 & 100 & 82.9 & 16.9 & 117 \\
\hline Mir-339 & 100 & 108 & 17.9 & 112 \\
\hline
\end{tabular}

Figure 4. A. Co-culture of chondrocytes in alginate beads (or osteoblasts in 2D culture) in standard medium (Gordeladze 2011). Osteochondral cells were differentiated from hMSCs in appropriate medium for 14 days. Then, a dentine slice, with close to 100\% enriched peripheral blood monocytes (PBMCs) had been differentiated for 14 days with RANKL-L and M-CSF, was placed adjacent to the osteochondral cells, and both cell types were co-cultured for 7 days. At the end of the experiment, the number of TRAP-positive cells was counted, and the eroded dentine surface (\%) was estimated in a reflecting light microscope. B. Co-culture of chondrocytes in alginate beads (or osteoblasts in 2D culture) in standard medium (Gordeladze 2011). The subject experimental setting was also applied to differentiating osteoblast. Here, we used the cytokine mixture as described in (B), and antago-microRNAs counteracting the os- 
teochondral signature. Variables analysed were much like those for the experiment with chondrocytes. However, here we measured mineralizing surface (Alizarin red S coloration) induced by the osteoblasts in a 2D culture.C. Co-culture of chondrocytes in alginate beads (or osteoblasts in 2D culture) in standard medium (Gordeladze 2011). The above mentioned experimental setting was used with chondrocytes differentiated from hMSCs in alginate beads in the absence or presence of cytokines (IL-1, IL-6, IL-17, and TNFa), pre-microRNAs of all microRNA species of the osteochondral signature, or the set of cytokines along with the pre-microRNAs. Parameters analysed were: Q-PCR of transcription factors (TFs) and marker genes expressed by the chondrocytes, clinical score of alginate beads containing chondrocytes embedded in newly synthesized proteoglycan matrix, the number of Trap-positive osteoclasts, and the eroded dentine surface (\%).

Termination: Sclerostin expression then fades or ceases, and the process of bone formation comes to a halt. The newly deposited osteoid is subsequently mineralized, the bone surfaces return to their resting state, This is when bone-lining cells intercalate with osteomacs, and the remodelling cycle is concluded (Raggatt and Partridge 2010).

\section{Osteoclast differentiation and activation}

\subsection{Introduction}

Rheumatoid arthritis (RA) is a chronic inflammatory disease perpetuated by a dysregulated immune system. The cause remains grossly unknown, however both environmental and genetic factors are believed to contribute to RA development (Kochi 2010; Boissier 2011). The disease is characterized by joint inflammation, initially resulting in pain and swelling, and in a majority of the patients, erosion of bone and cartilage takes place (Lories 2011). The goal of current treatment regimens is to control inflammation, as well as to halt or retard the progression of damage of the bone structure of the affected joint(s), as measured by X-ray analysis (Tan and Conaghan 2011). The RA joint, synovial fluid, and synovium contain a large variety of hematopoietic cells, which are situated in direct proximity to the articular cartilage and its underlying bone structures (Carvalheiro, da Silva et al. 2012; Shegarfi, Naddafi et al. 2012). Hence, the synovial fluid with its contents of immune cells and cytokines secreted from the cells in question, undoubtedly are responsible for the joint-destructive process (Iwamoto, Okamoto et al. 2008; Yamada, Nakashima et al. 2008).

\subsection{Osteoclast differentiation}

Physiological and pathological bone resorption is achieved by the osteoclast. Osteoclasts are large 20 to $100 \mu \mathrm{m}$ multinucleated cells containing from three to 100 nuclei with many mitochondria, lysosomes, dense granules, vesicles, and an extensive Golgi network required for the synthesis and secretion of factors required to degrade bone matrix and subsequently phagocytose the resorbed products (Holtrop and King 1977). Tartrate-resistant acid phosphatase (TRAP) (Minkin 1982), cathepsin K (Drake, Dodds et al. 1996), the calcitonin (CT) receptor (Hattersley and Chambers 1989), as well as $\alpha v \beta 3$ integrin (Davies, Warwick et al. 1989) are marker gene products of the mature osteoclast (Teitelbaum 2000). The initial event in bone resorption is the attachment of the mature osteoclast to the bone matrix. Cell surface $\alpha \mathrm{v} \beta 3$ integrins bind to a plethora of extracellular matrix proteins synthesized and secreted by 
osteoblastic cells, including vitronectin, osteopontin, and bone sialoprotein. Arg-Gly-Aspcontaining peptides, Arg-Gly-Asp mimetics, and blocking antibodies to $\alpha v \beta 3$ integrins have been shown to inhibit bone resorption, both in vitro and in vivo, indicating that this integrin plays a pivotal role in osteoclast functioning (Horton, Taylor et al. 1991). Once attached to bone, the osteoclast generates an isolated extracellular, acidic microenvironment between itself and the bone surface by creating a sealing zone structure unique to the osteoclast.

\subsection{Osteoclast activation}

As described in a previous paragraph, macrophage CSF (M-CSF) and receptor activator of NFkB ligand (RANK-L) are the most important factors known to date to drive osteoclast formation and activity (Lacey, Timms et al. 1998; Quinn, Elliott et al. 1998). M-CSF serves as a survival factor for osteoclast precursors due to up-regulation of Bcl-XL, inhibition of caspase-9 activation (Woo, Kim et al. 2002), and the support of mature osteoclast survival by preventing apoptosis (Fuller, Owens et al. 1993). Exposure to M-CSF also stimulates receptor activator of NFkB (RANK) expression in osteoclast precursor cells, thereby allowing RANK-L to drive formation of mature osteoclasts (Arai, Miyamoto et al. 1999). RANK-L is a trans-membrane protein expressed by activated osteoblasts, synovial fibroblasts, as well as T-cells. It can also be proteolytically cleaved by TNF convertase (TACE) to generate a soluble molecule that has osteoclastic activity at distal sites (Hofbauer, Khosla et al. 2000; Li, Toraldo et al. 2007). RANK$\mathrm{L}$ induced osteoclastogenesis is inhibited by OPG (osteoprotegerin), a soluble decoy receptor for RANKL, which is also produced by a variety of cells including osteoblasts, synovial fibroblasts, B-cells, and T-cells (Simonet, Lacey et al. 1997; Hofbauer, Khosla et al. 2000; Takayanagi, Iizuka et al. 2000; Gillespie 2007; Li, Toraldo et al. 2007). In the arthritic joint, there is a significant macrophage infiltrate, and the extent of the subject infiltration correlates heavily with the extent of joint erosion in both RA patients and animal models with arthritis (Yanni, Whelan et al. 1994). Synovial macrophages isolated from different types of arthritis may differentiate in vitro to fully functional osteoclasts subsequent to RANK-L stimulation as well as independently of the RANK/RANK-L signalling pathway, i.e. after short term exposure to TNF $\alpha$ and IL-1 $\alpha$ (Adamopoulos, Sabokbar et al. 2006). Stimulatory, co-stimulatory, and/or inhibitory signals may be provided by adjacent T-cell species present in the inflammatory infiltrate.

\section{T-cell subsets and their action on osteoclasts}

\subsection{Th17 cells versus Th1 and Th2 cells}

The Th17 lineage has only recently been fully characterized (Adamopoulos and Bowman 2008) and the factors involved in its differentiation are still being identified and sorted out (Hirota, Hashimoto et al. 2007; Parsonage, Filer et al. 2008). When a (CD4+) naïve T cell is activated in the presence of transforming growth factor beta (TGF $\beta$ ) plus IL- 6 in the mouse or TGF $\beta$ plus an inflammatory stimulus in the human, the resulting clonal memory T-cell population will be instructed to produce the Th17 signature cytokines IL-17A, IL-17F, IL-22, 
and (only in humans) IL-26 (Manel, Unutmaz et al. 2008). The inflammatory stimuli in the human setting can be IL-1 $\beta$, IL-6, IL-21, and/or IL-23 (Langrish, Chen et al. 2005; AcostaRodriguez, Napolitani et al. 2007; Wilson, Boniface et al. 2007; Manel, Unutmaz et al. 2008; Volpe, Servant et al. 2008; Yang, Anderson et al. 2008). IL-17A is the only Th17 signature cytokine presently known to impact osteoclast biology. Synovial residing and synovial fluid derived macrophages are able to differentiate to fully functional bone-resorbing osteoclasts, and Th17- induced synovial macrophage-mediated osteoclast differentiation is believed to represent an important mechanism in bone destruction associated with rheumatoid arthritis (Fujikawa, Sabokbar et al. 1996; Adamopoulos, Sabokbar et al. 2006). Several types of IL-17A antagonists have been applied in a variety of experimental arthritis models to elucidate the efficacy of therapeutic IL-17A neutralization. Polyclonal anti-IL-17A antibody treatment after disease induction in the collagen induced arthritis (CIA) model diminished clinical scores subsequent to 10 days of therapy, compared with controls. Ankle and knee joints displayed reduced synovitis, cartilage destruction, chondrocyte cell death, depletion of proteoglycans, and bone erosion (estimated by both histology and X-ray analyses) (Lubberts, Koenders et al. 2004). Polyclonal anti-IL-17A antibodies also suppressed exacerbation of antigen-induced knee swelling, proteoglycan depletion, and bone erosion in experimental RA model systems upon reintroduction of the antigen (Koenders, Lubberts et al. 2005). The Th1 and Th2 cell lineages, of which the Th1 cell phenotype resembles that of Th17 cells, have interestingly proven to counteract the effect of Th17 cells on osteoclastogenesis, both in vitro and in experimental and idiopathic conditions of rheumatoid arthritis (Aarvak, Chabaud et al. 1999).

\subsection{T-helper 17 cells in Rheumatoid Arthritis (RA)}

A multitude of experimental reports point to Th17 cell mediated inflammation being associated with RA. IL-17A protein is located in both the synovium and the synovial fluid of patients suffering from the disease. It was also demonstrated that a subset of T-cell lines could be expanded in vitro from their RA synovium, which expresses both IL-17A and IFN $\gamma$ [(Aarvak, Chabaud et al. 1999; Pene, Chevalier et al. 2008). Classical IFN $\gamma$-only Th1 cells were also present, constituting a clonal species which can be expanded from the RA synovium (Aarvak, Chabaud et al. 1999; Yamada, Nakashima et al. 2008). Exploratory medical studies, however, not only establish the association of IL-17A with disease development, but also implicate IL-17A with poor disease prognosis. The IL-17A message in synovial membrane biopsies was one factor (including TNF, IL-1 $\beta$, and IL-10) being predictive for subsequent bone erosion and joint damage, as assessed by NMR imaging and X-ray analyses (Kirkham, Lassere et al. 2006). Taken together, these data support the notion that IL-17A is present in the inflamed synovium and that the levels of IL-17A expression correlate with poor prognosis and greater joint destruction. All in all, Th17 cells secrete master factors that can directly and indirectly drive osteoclast differentiation and activation. It is, however, a consensus in the field of T-cell research that signature cytokines of the Th1, Th2, and Treg lineages inhibit Th17 cell development. Therefore, one has construed that osteoclastogenesis is inhibited by the same Th1, Th2, and Treg lineage signature cytokines counteracting the stimulatory potential of the cytokine mixture secreted by the Th17 cells (Adamopoulos, Sabokbar et al. 2006; Adamopoulos and Bowman 2008). Contrastingly, when comparing clonal cells lines, 
Th1 cells may resemble Th17 cells, questioning the postulate that the Th1 cell lineage consistently will counteract the impact of Th17 cells. In some instances, infiltration by Th1 cells may even potentiate the detrimental effect of Th17 cells on the cartilage and bone in affected joints (Yssel et al., unpublished data).

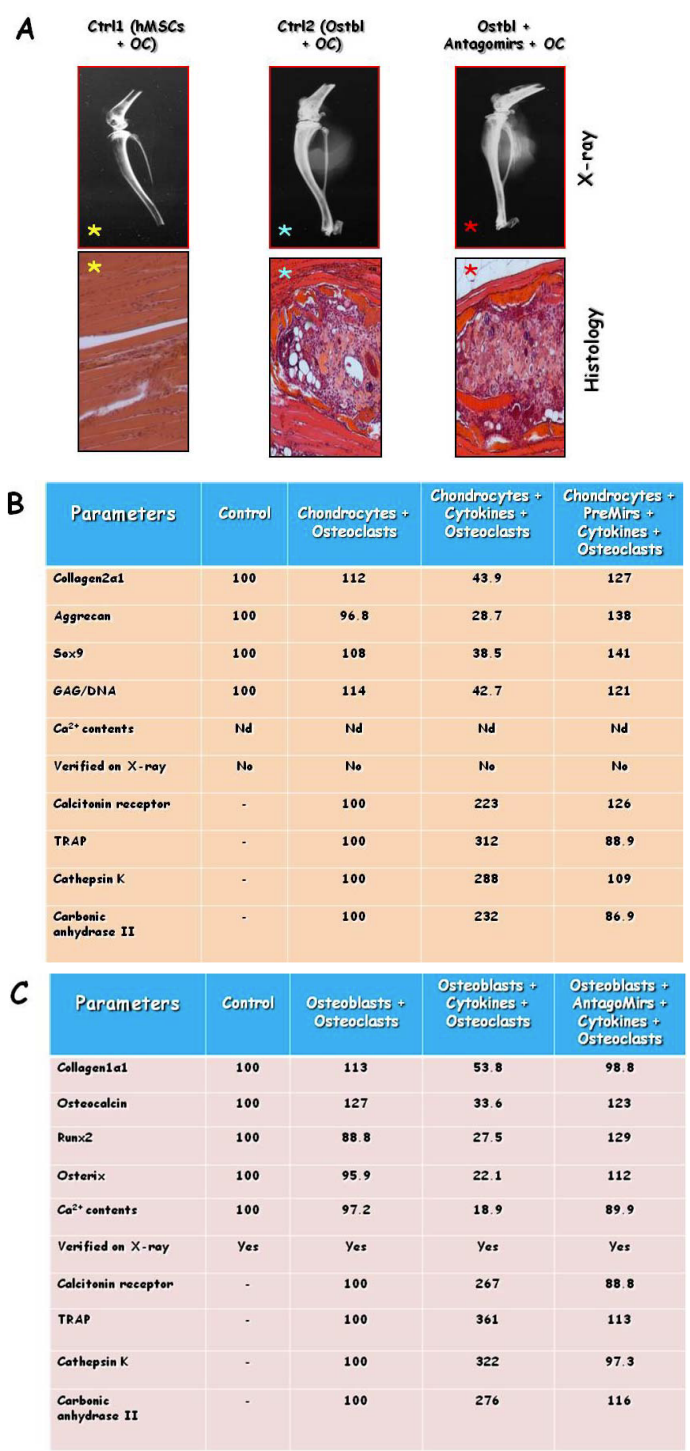

Figure 5. A. Effect of microRNA manipulations and cytokines on human osteochondral cells and osteoclasts injected into the tibial muscle of SCID mice. X-ray and tissue histology of the lower leg ( $m$. tibialis) from SCID mice. Each tibial muscle was injected with hMSCs (2.5 million cells) and osteoclasts ( 0.5 million cells). Yellow, blue and red stars indicate 
that X-ray analyses and histology (visual parameters) were not significantly altered when cells were manipulated by cytokines (IL-1, IL-6, IL-17, and TNFa). However, molecular parameters were dramatically changed. X-ray analyses and histology were performed on excised legs 21 days subsequent to cell deposition. B. Differentiated chondrocytes (21 days) exposed to cytokines (initial 14 days) or pre-microRNAs (initial 14 days) and osteoclasts injected into the tibial muscle of SCID mice. Molecular markers of chondrocytes and osteoclasts (PBMCs differentiated for 10 days) were analysed by Q-PCR or colorimetric methods (GAG = glucosaminoglycan/DNA). Nd = Not determined. All values are given relative to control conditions (chondrocytes alone or chondrocytes + osteoclasts). C. Differentiated osteoblasts (21 days) exposed to cytokines (initial 14 days) or antago-microRNAs (initial 14 days) and osteoclasts were injected into the tibial muscle of SCID mice. Molecular markers of osteoblasts and osteoclasts (PBMCs differentiated for 10 days) were analysed by Q-PCR or colorimetric methods (Ca2+-analyses in $\mathrm{HCl}$-extracts). All values are given related to control conditions (osteoblasts alone or osteoblasts + osteoclasts).

\section{Cell-to-cell communication through exosomes}

Cell-to-cell communication is required to guarantee proper coordination among different cell types within tissues. Cells may communicate by soluble factors (Majka, Janowska-Wieczorek et al. 2001), adhesion molecule mediated cell-to-cell interactions including cytonemes connecting neighbouring cells enabling ligand-receptor-mediated transfer of surface-associated molecules, or by tunnelling nanotubules which establish conduits between cells. These would allow the transfer of both surface molecules and cytoplasmic components (Rustom, Saffrich et al. 2004; Sherer and Mothes 2008). Recently published studies have suggested that cells may also communicate by spherical membrane fragments called micro-vesicles (MVs) or exosomes (Ratajczak, Wysoczynski et al. 2006). MVs or exosomes were since long considered to be inert cellular debris, and the frequently observed vesicles by electron microscopy in the interstitial space of tissues or in blood were considered the consequence of cell damage or a result of dynamic plasma membrane turnover (Siekevitz 1972). It was suggested that the circular plasma membrane fragments released from human cells might possibly result from a specific process, and it was shown that they carried functional membrane enzymes in the same ratio as the membrane of the cells they originated from (De Broe, Wieme et al. 1977). However, only a few recent studies have assigned a defined function to the vesicles/exosomes released to the microenvironment by various cell types. Two distinct processes of shedding vesicles from the cells have been described. MVs may originate from the endosomal membrane compartment that, subsequent to fusion with the plasma membrane, is extruded from the cell surface of activated cells as exosomes (Heijnen, Schiel et al. 1999; Rozmyslowicz, Majka et al. 2003). Otherwise, MVs may take origin by direct budding from the cell plasma membrane as shedding vesicles (Cocucci, Racchetti et al. 2009).

Released MVs may remain in the extracellular space in the proximity of the place of origin, or they may enter into the circulation. One has hypothesized that, under normal healthy conditions and disease states, micro-vesicles contain microRNAs, contributing to biological homeostasis in general (Hunter, Ismail et al. 2008; Gordeladze, Djouad et al. 2009; Gordeladze 2011). It may also be hypothesized that MVs or exosomes, when present in disproportional amounts within a tissue during development of certain diseases, in fact may affect all adjacent cell types, thus altering their phenotypes and main functional properties (Camussi, Deregibus et al. 2010). 


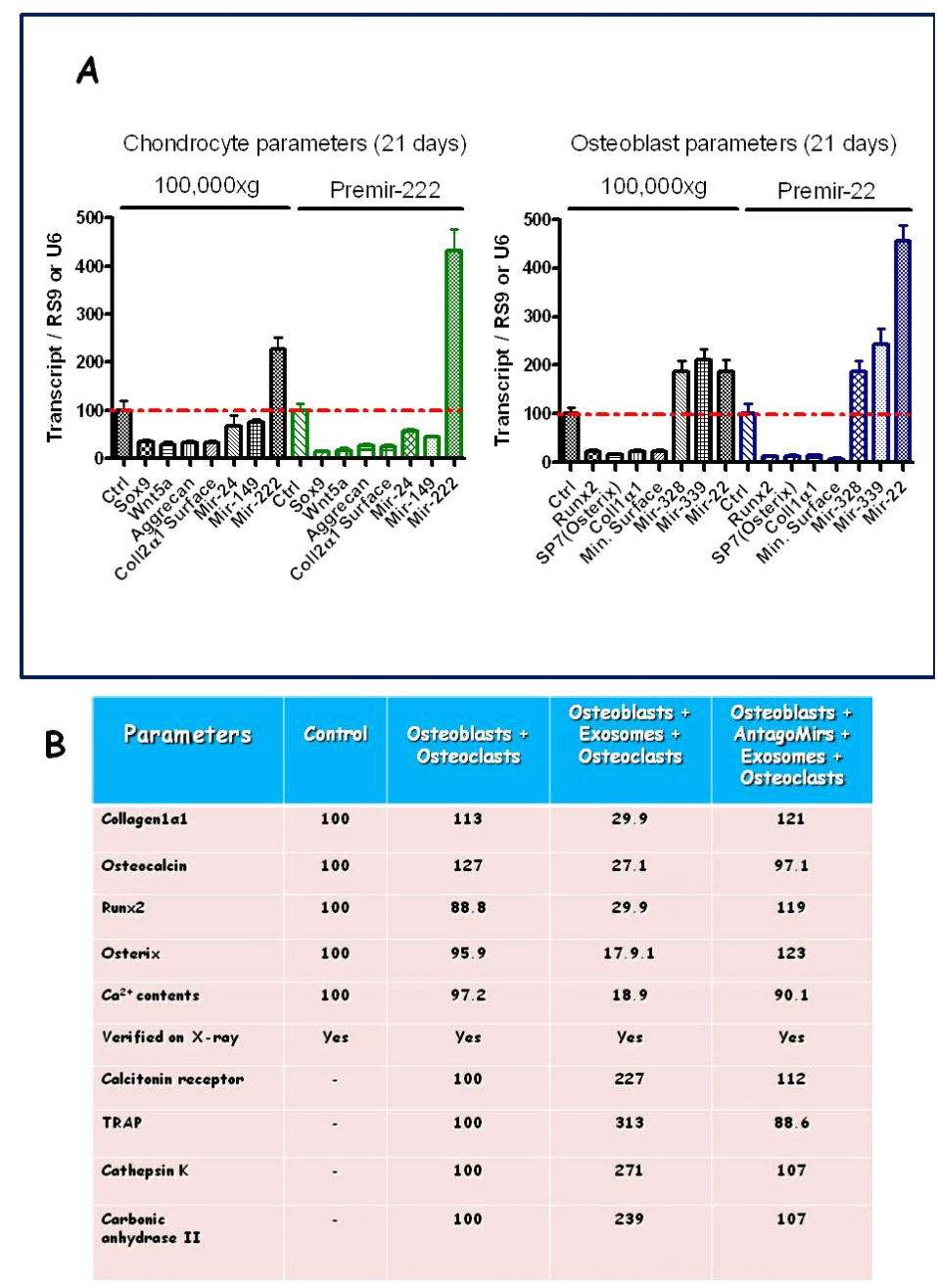

Figure 6. A. The effect of exosomes-containing 100,000xg fractions of supernatants derived from activated human Th17 cells on human chondrocytes and osteoblasts in the presence and absence of osteoclasts. Chondrocytes (500,000 cells) derived from hMSCs were either exposed to $1 \mathrm{ml}$ volume containing exosomes from 250,000 Th-17 cells or pre-microRNA 222 (which is highly expressed in Th17 cells, but not in chondrocytes) and analysed for marker gene and microRNA expressions using standard Q-PCR methods (Ambion) or immunohistochemistry (aggrecan and collagen $2 a 1$ positive surfaces). Osteoblasts (500,000 cells) derived from hMSCs were either exposed to $1 \mathrm{ml}$ volume containing exosomes from 250,000 Th-17 cells or pre-microRNA 22 (which is highly expressed in Th-17 cells, but not in 
osteoblasts) and analysed for marker gene and microRNA expressions using standard Q-PCR methods (Ambion) or colorimetric methods (mineralized surface visualized by Alizarin red S). B. The effect of exosomes-containing 100,000xg fractions of supernatants derived from activated human Th17 cells on human chondrocytes and osteoblasts in the presence and absence of osteoclasts. Differentiated osteoblasts ( 21 days) exposed to exosomes from Th17 cells (initial 14 days) or antago-microRNAs (initial 14 days) and osteoclasts were injected into the tibial muscle of SCID mice, and molecular markers of osteoblasts and osteoclasts (PBMCs differentiated for 10 days) were analysed by Q-PCR or colorimetric methods ( $\mathrm{Ca}^{2+}$-analyses in $\mathrm{HCl}$-extracts). All values are given related to control conditions (osteoblasts alone or osteoblasts + osteoclasts).

In one publication, it was shown that exosomes from healthy volunteers matched mononuclear cells and contained 420 known mature microRNAs (Hunter, Ismail et al. 2008). Hierarchical clustering of the data sets pointed to significant differences in microRNA expression between peripheral blood mononuclear cells (PBMCs) and plasma micro-vesicles. It was observed that 71 microRNAs co-expressed between micro-vesicles and PBMCs. Prediction of the gene targets and associated biological pathways regulated by the detected microRNAs demonstrated that the majority of these microRNAs expressed in the micro-vesicles from the blood were predicted to regulate the differentiation of blood cells and their metabolic pathways (Hunter, Ismail et al. 2008). Interestingly, a small group of these microRNA species were also predicted to serve as important modulators of immune function. The microRNAs in question were hypothesized to be taken up by adjacent cells, and thus it may be asserted that exosomes from Th17 cells, known to be present in vast numbers in articular fluid of patients with rheumatoid arthritis (Adamopoulos and Bowman 2008; Nakashima and Takayanagi 2009; Raggatt and Partridge 2010; Nakashima, Hayashi et al. 2012) with rheumatoid arthritis, may disturb the phenotypic characteristics of osteochondral cells in inflamed joints.

\section{Results and discussion}

\subsection{How does the osteochondral microRNA profile work?}

In Fig. 1, we have presented a microRNA signature, consisting of six microRNA species. These species have been selected amongst microRNAs putatively targeting one or several of 14 transcription factors (TFs) deemed important for osteoblast differentiation from stem cells or precursor cells (Gordeladze, Djouad et al. 2009). It was shown through reporter constructs, that nine of these 14 TFs were targeted by the six microRNAs. Literature studies further indicated that mir-149, and possibly also mir-24, may serve as a switch mechanism instrumental in determining whether stem cells will become osteoblasts or chondrocytes by interfering with activators, inhibitors and associated proteins instrumental in modulating the effects of Sox9 and Runx2 (lee legend to Fig. 1). These two TFs oppose each other when it comes to differentiation of stem cells or progenitor cells to become mature osteoblasts and chondrocytes (Marie 2008; Gordeladze, Djouad et al. 2009).

A bioinformatics study (see Fig. 2, A-C), using the Mir@nt@n algorithm (Le Bechec, PortalesCasamar et al. 2011), showed that the microRNA species mir-149, mir-328, and mir-339 in particular seemed to serve important regulatory roles when looking at some important criteria for osteoblastogenesis. (A) JUN = transcription factor AP-1, known to be mandatory for 
osteoblast differentiation (Gordeladze, Djouad et al., 2009), appears to exert a major impact on mir-339, and mir-149 in a hierarchical feed-forward fashion. (B) ETS1 and SP3, two of the 14 TFs constituting the selection criteria for the osteochondral microRNA profile (Gordeladze, Reseland et al., 2011), appeared to serve as inductors of several microRNAs, among which we find the microRNA species mir-149, mir-328, and mir-339. However, ETS1 and SP3 (along with SP1 and SP7 = Osterix) are up-regulated in osteoblastic cells. Hence, the positive control exerted by ETS1 and SP3 may be counterproductive, or maybe not? (C) In a more complicated hierarchical feed-forward network, it can be noted that Sox9, an important regulator of chondrogenesis, actually serves as a master regulator of mir-149 and mir-328, which both are up-regulated in chondrocytes. When modelling the osteochondral microRNA profile with all know factors of the canonical WNT-pathway (using all factors from the KEGG's pathway), a stunningly close interaction between some of the microRNA species of the profile and factors discerning the osteoblast from the chondrocyte phenotypes emerged.

It may therefore be postulated that the presently described osteochondral microRNA profile serves as a discriminator between osteoblasts and chondrocytes, and can be used as such in the process of cell engineering, while the microRNAs published as markers for osteoblasts by G. Stein and his collaborators distinguish osteoblasts from all other cell phenotypes in a comprehensive manner (Li, Hassan et al. 2008; Hassan, Gordon et al. 2010; Zhang, Xie et al. 2011).

\subsection{Summary of experiments proving the functionality of the osteochondral microRNA profile}

In Fig. 3, we list the results of some experiments where the endogenous levels of each microRNA species of the profile are altered. These experiments indicate that chondrocytes show optimal microRNA profile levels, since some 6-8 fold increase do not alter their characteristics, subsequent to differentiation from hMSCs. A reduction to 12-14\% of their endogenous levels shows that mir-16, mir-125b, mir-328, and mir-339 are necessary for chondrogenesis, while mir-24 and mir-149 are not. Contrastingly, enhancing the endogenous levels of all the microRNA species in hMSCs more or less block their differentiation towards the osteoblast lineage. As expected, reducing their levels in hMSCs by a factor of 2-2.5 clearly fortified their phenotype as osteoblasts. This experiment establishes the six microRNAs as instrumental in osteochondral differentiation, while mir-24 and mir-149 play a dual role, being able to simultaneously block osteoblastogenesis and stimulate chondrogenesis. An explanation for this dual role has been sought in the information conveyed in Figs. 3A\&B, looking for published data on their known targets, as well as bioinformatics profiles, i.e. positions in feed-forward interaction networks (see legend to Fig. 3).

\subsection{Osteochondral cells influence osteoclasts: The impact of cytokines}

In Fig. 4A, we depict a co-culture system where hMSCs are differentiated towards osteochondral cells for 2 weeks and co-cultured with differentiated osteoclasts on dentine for a third week. The osteoclasts have been differentiated from hPBMCs for 1 week with RANK-L and 
M-CSF. It is clear that chondrocytes do stimulate osteoclasts to resorb bone, and that exposure of the chondrocytes to cytokines ("artificial" synovial fluid from individuals with rheumatoid arthritis $=$ RA, i.e. growth medium containing IL-1, IL-6, IL-17A and TNF $\alpha$ ) enhances the osteoclasts' bone resorbing potential.

\subsection{MicroRNA manipulation of osteochondral cells: Obliteration of the impact of cytokines}

In Figs. 4B\&C it is shown that TGF $\beta_{3}$-stimulated hMSCs to chondrocytes or BMP2-stimulated hMSCs to osteoblasts exposed to cytokines in fact are protected against loss of phenotypes and normalize (i.e. reduce) their ability to enhance osteoblast activity upon normalization of their endogenous microRNA profile. The tables show data for Q-PCR of phenotype marker genes, RANK-L and OPG (counteracting each other), functional markers like clinical score (constituted by chondrocyte bead histology, immunohistochemistry, distance between cells, GAGcontents), mineralizing surface, percentage of TRAP-positive cells containing $\geq 3$ nuclei, eroded dentine surface (\%), as well as Q-PCR of microRNAs of the osteochondral signature.

\subsection{In vivo model of osteochondral cells interacting with osteoclasts}

In Fig 5A, X-ray pictures and biopsy histology of tibial muscles of SCID mice are shown. Their muscle tissue has been injected with hMSCs, osteoblasts without and with the presence of differentiated osteoclasts. The osteoblasts were either loaded with antago-microRNAs of the osteochondral signature or not. Additional experiments where hMSCs or osteoblasts had been exposed to cytokines alone or cytokines and antago-microRNAs together are not shown on the figure. A similar experimental matrix was conducted (but not shown) with chondrocytes differentiated from hMSCs. The pictures indicate that mature osteoblasts and osteoclasts are able to build bone within the muscle tissue, and that a suppression of the endogenous levels of the microRNA species of the osteochondral signature lead to enhanced mineralization of osteoid produced.

When chondrocytes (see Fig. 5B) are pre-exposed to osteoblasts, it appears that the cells are able to maintain their net cartilage-building potential, possibly by down-regulating their cytokine-induced osteoclast-activating potential. The same phenomenon is also seen when osteoblasts are injected into the $m$. tibialis of SCID mice.

Cytokines induce a reduction in osteoblast functions, while also enhancing osteoclast activation resulting in a lessened bone deposit, while introduction of antago-microRNAs reverses the action of cytokines towards maintenance of the bone-building capacity of the osteoblast (see Fig. 5C). A special attention to the Q-PCR data on osteoclast specific markers (CT receptor, TRAP, cathepsin K and CA II) analysed in the excised de novo bone tissue is warranted. Furthermore, it should be emphasized that the X-ray analyses of the lower legs injected with osteoblasts were corroborated analysing the $\mathrm{Ca}^{2+}$-contents in acid extracts/digests, using standard spectrophotometry. 


\subsection{The effect of exosomes derived from activated Th17 cells}

Lastly, we shall address the hypothesis put forward that exosomes shedded from immune cells may affect the characteristics of osteochondral cells. Th17 cell exosomes-like particles were obtained from 100,000xg centrifugates of cultures of activated Th17 cells (Larsen, Arnaud et al. 2011). The pellets were examined for microRNAs (micro-arrays), and 55 microRNA-species were identified. Of these, hsa-mir-22- and hsa-mir-222 are especially interesting, since the exosome-fraction contained large quantities of these, and because Th17 cell clones also produced large quantities of the same microRNA species, and since osteoblasts and chondrocytes expressed relatively low levels of the mir-22, and mir-222 species, respectively.

Fig. 6A depicts chondrocytes having been differentiated from hMSCs for 2 weeks receiving control medium or control medium + Th17 cells derived exosomes or pre-microRNA mir-222 on days 15-21. Q-PCR of marker genes and microRNA transcripts, as well as collagen2 $\alpha 1-$ positive surface analyses were conducted and showed that chondrocyte characteristics were suppressed. The same experiment was conducted on hMSCs differentiated towards osteoblasts in the absence or presence of Th17 cell exosomes or pre-microRNA mir-22, and phenotype marker analyses (i.e. Q-PCR of marker gene transcripts and mineralized surface) conducted. Not surprisingly, the osteoblast phenotype was suppressed due to both treatments. This indicates that exosomes from Th17 cells contain microRNAs detrimental to the functioning of mature chondrocytes and osteoblasts, and it may well be so that engineered osteochondral cells, deposited within a joint to replace damaged tissues, should be made resilient to inflammatory cells to maintain their proper phenotypic functioning over time.

One experiment (see Fig. 6B) with osteoblasts, osteoclasts, exosomes and antago-microRNAs of the osteochondral signature was performed on SCID mice, as envisaged earlier. X-rays (not shown) and analyses on excised biopsies from the tibial muscle showed that exposure to Th17 cell exosomes hampered the development of the matrix-producing and mineralizing properties of osteoblasts, while enhancing activating of adjacent osteoclasts. The introduction of antago-microRNAs of the osteochondral signature did, however, reinstall the osteoblasts' ability to produce bone, which was not degraded by osteoclasts. This phenomenon was corroborated through analyses of the $\mathrm{Ca}^{2+}$-contents of the biopsies taken (see also text-Fig. 5B).

\subsection{Other results obtained}

The present series of experiments also addressed whether certain scaffold materials and their 3D-structures were better suited to produce osteochondral cells with a "true" (in vivo like) phenotype being more robust and resilient towards provocations, such as chronic disease states. Whether we used scaffolds, i.e. alginate beads instead of micro-pellets, or hydroxyapatite instead of 2D-growth, really did not matter. However, we did not use promising scaffolds for osteoblast differentiation, like $\mathrm{TiO}_{2}$ or surfaces with an inverted profile of cellular outer membrane surface nanostructures (Muys, Alkaisi et al. 2006; De Smet, Jaecques et al. 2007; Mazzola, Bemporad et al. 2011). 


\subsection{General discussion of the findings presented}

This report is more about experimental approaches and results speaking for themselves than a lengthy discussion of their implications from a theoretical point of view. A few issues do, nevertheless, deserve attention.

Most reports on microRNA impact on cell differentiation and phenotype development and stability focus on one microRNA species only, or they present a microRNA micro-array showing a bulk of microRNAs being up- and down-regulated subsequent to cell differentiation. Since microRNAs exert different binding kinetics when they bind to different mRNAs at various places (not only to the 3'-UTR, but also to the 5'-UTR region) (Moretti, Thermann et al. 2010), it is reasonable to believe that a minimum effective group of microRNAs is necessary and sufficient to ensure complete differentiation and stability of a certain cell phenotype (Gordeladze, Djouad et al. 2009; Iorio, Casalini et al. 2011; Schoof, Botelho et al. 2012). The osteochondral signature of microRNAs presented in this chapter may serve as such a group, discriminating between cell types, however, several other microRNA species described by G. Stein and collaborators (Li, Hassan et al. 2009; Hassan, Gordon et al. 2010; Zhang, Xie et al. 2011) may serve the same purpose. In fact, our microRNA micro-arrays obtained from osteoblastic cells in different stages of differentiation from hMSCs do overlap extensively to the ones published in recent literature (Lian, Javed et al. 2004).

Which microRNAs are to be construed as the "true" marker for osteoblasts? They may constitute more than the six species presented here, or even other than the ones we have focussed on. The main issue is how they interact with feed-forward and feed-back regulatory loops, in which transcription factors and marker genes are positioned. This issue has been addressed in the bioinformatics exercises outlined in Fig. 2. Here, mir-26b pops up as being important, and this microRNA is known to a network of factors serving as a possible target for RNA-based therapy of bone diseases (Luzi, Marini et al. 2012). The main issue is: which microRNAs out of a smaller group will be necessary and sufficient to determine or alter a certain phenotype? Our experiments with the present osteochondral microRNA signature seem to render one such microRNA group distinguishing between osteoblasts and chondrocytes. A further proof of concept put forward is the fact that these microRNAs can be applied to trans-differentiate osteoblastic cells to chondrocytes and back again, and they are also able to a large extent (some 85\%) substitute growth factors (TGFs and BMPs) as mandatory to obtain chondrocytes and osteoblasts from hMSCs or human adipose tissue derived stem cells (hADMSCs) (Gordeladze, JO, unpublished results).

In chapter 2 of the book "Regenerative Medicine and Tissue Engineering: Cells and BioMaterials" (Gordeladze 2011), we introduced a concept of compatibility score between microRNA micro-arrays and transcriptomes obtained from chondrocytes and osteoblasts differentiated from precursor cells of stem cell origin (ref). We still believe that a proper way to ensure "correct" phenotype is to correlate consensus micro-arrays of marker gene transcripts with microRNA micro-arrays; however, such analyses were not performed on the present manipulated biological material. Due to relative meticulous experimentation consuming a lot of 
manpower, biological material and costly analyses, it was not performed. However, microarrays analysed at certain intervals during a differentiation process may seem necessary to be able to arrive at the superior microRNA profiles describing a certain cell phenotype to be distinguished from other phenotypes.

One question pertaining to the assurance of a reproducible process of establishing engineered cells with the "correct" phenotype is: Why play with microRNAs, is it not enough to manipulate using growth factors and the 3D-structure, in which the precursor cells are grown? Firstly, it is important to know the interplay between factors able to induce a certain cell phenotype and/or trans-differentiate phenotypes. In this respect, it seems fruitful to concentrate on several microRNAs, and not only on one microRNA species. But, it is also reasonable to play with the expression of certain transcription factors (TFs) being positioned in key places in a regulatory network constituted by TFs, activators, inhibitors or associated proteins, as well as functional marker genes. Sox9, a TF deemed sufficient and necessary for chondrocyte development (see legend to Fig. 2C) may be over-expressed or suppressed to engineer chondrocytes or osteoblasts, since it affects the expression of important microRNAs and marker genes in a hierarchical fashion. Apparently, so do ETS1 and SP3, or MYC and JUN (see legend to Figs. 2B\&A).

However, it seems reasonable to believe that, at least during a shorter, critical period of time, externally added growth factors and the selection of biologically "correct" scaffold structures and materials will aid in the process of proper phenotype acquisition for tissue replacement purposes. It has also been pointed out that it is vital, for a proper tissue to be generated, to use cell material which can differentiate into several cell types constituting a certain tissue, or that the engineered cell material is able to recruit cells from the ambiance to produce a correctly functioning tissue in vivo. A good example of this seems to be an artificially made tracheal epithelium, using collagen vitrigel-sponge scaffolds containing bFGF as growth factor and chemotactic agent (Tani, Tada et al. 2012).

We are now seeking to produce polycistronic constructs containing pre-microRNAs or antago-microRNAs to be temporarily activated in order to test whether the microRNAbased concept of cell engineering may function in an in vivo animal model of rheumatoid arthritis, where articular joints are inflamed and contain immune cells (i.e. Th17 cells). Hence, we will be able to find out whether osteochondral cells may be engineered to withstand the detrimental effect of cytokines and exosomes containing (amongst many bioactive molecules) unwanted microRNA species. Promising small animal models to start with are collagen-induced arthritis in the mouse, and the senescence mouse (Aigner, Rose et al. 2004; Manolagas and Almeida 2007) prior to the testing in larger animals (i.e. sheep model systems) (Gordeladze, Reseland et al. 2009).

Lastly, the present experiments confirm the complexity of bone remodelling in the healthy individual and in patients with diseases affecting cartilage and bone structure. Not only hormones, like active vitamin D (calcitriol) and PTH affect bone turnover, via their dual effects on osteoblasts and osteoclasts. The interaction with the immune system directly interfering with the balance between RANK-L and OPG (Adamopoulos, Sabokbar et al. 2006; Nakashima, 
Hayashi et al. 2012), as well as cell-to-cell communication through exosomes, containing a plethora of bioactive molecules, urges new research approaches to unravel the intricate mechanisms ensuring bone and cartilage health before and after the onset of disease states damaging the tissues in question. We have here confirmed that chondrocytes, in fact, do affect osteoclasts directly (Xiong, Onal et al. 2011), and that cytokines obtained from Th-cells, and Th17 cells in particular, are detrimental to the osteochondral phenotypes, with an additional activation of osteoclasts.

The present experimental setting also show that exosomes from Th17 cells may interfere with both the chondrocytic and osteoblastic phenotypes in a negative fashion (i.e. phenotype acquisition and matrix deposition), while also speeding up bone remodelling via overactivation of osteoclasts embedded in the bone adjacent to the cartilage lining. These findings are consistent with the development of cartilage loss and the appearance of osteophytes (newly formed bone in disarray) in arthritic joints due to the progressing autoimmune process characteristic of rheumatoid arthritis (Hayashi, Xu et al. 2012).

\section{Summary and future prospects}

This particular report entails the use of transient microRNA manipulations to ensure acquisition of proper osteochondral phenotypes when engineering cells to replace damaged bone and cartilage in patients with inflammatory diseases targeting articular joints (in particular rheumatoid arthritis). We have shown that cell engineering, as a research field, needs to take into consideration how osteochondral cells affect osteoclasts directly, and that osteochondral cells may lose their acquired phenotypes upon exposure to cytokines (e.g. IL-1, IL-6, IL-17, and $\mathrm{TNF} \alpha$ ) or micro-RNA-containing exosome-like particles derived from activated Th17 cells. These detrimental effects can be counteracted by manipulating stem cell microRNA contents (the optimal minimal number and species of microRNAs are yet to be defined).

When refining the search for the minimal number of effective microRNAs, it is recommended that bioinformatics approaches are used along with micro-RNA micro-arrays and marker gene transcriptomes in engineered osteochondral cells, and that maximal compatibility score (Gordeladze 2011) between them are obtained. Assessment of phenotypes obtained should include analyses of how and to which extent these cells affect osteoclasts, and whether altered (i.e. enhanced) remodelling of bone formed within an in vivo model system (e.g. calcium deposits in the tibial muscle of SCID mice) of choice.

\section{Acknowledgements}

This project was supported by EU FP6 integrated project "Genostem cells engineering for connective tissue disorders", Norwegian Center for Stem Cell research, The National Hospital, Oslo, Norway, and The Research Council of Norway. 


\section{Author details}

Jan O. Gordeladze ${ }^{1,2^{*}}$, Janne E. Reseland ${ }^{3}$, Tommy A. Karlsen ${ }^{1,2}$, Rune B. Jakobsen ${ }^{2}$, Astrid K. Stunes ${ }^{4}$, Unni Syversen ${ }^{4}$, Lars Engebretsen ${ }^{5,6}$, Ståle P. Lyngstadaas ${ }^{3}$ and Christian Jorgensen ${ }^{7,8}$

*Address all correspondence to: j.o.gordeladze@medisin.uio.no

1 Department of Medical Biochemistry, Institute of Basic Medical Science, University of Oslo, Norway

2 Norwegian Center for Stem Cell research, The National Hospital, Oslo, Norway

3 Department of Biomaterials, Faculty of Dentistry, University of Oslo, Norway

4 Department of Cancer Research and Molecular Medicine, Norwegian University of Science and Technology, Trondheim, Norway

5 Orthopaedic Center, Ullevål University Hospital, Oslo, Norway

6 Faculty of medicine, University of Oslo, Norway

7 INSERM U844, Montpellier, France

8 Unité Immuno-Rhumatologie Thérapeutique, Centre Hospitalier Universitaire (CHU) Lapeyronie, Montpellier, France

\section{References}

[1] Acosta-rodriguez, E. V, Napolitani, G, et al. (2007). Interleukins 1beta and 6 but not transforming growth factor-beta are essential for the differentiation of interleukin 17producing human T helper cells." Nat Immunol , 8(9), 942-949.

[2] Adamopoulos, I. E, \& Bowman, E. P. (2008). Immune regulation of bone loss by Th17 cells." Arthritis Res Ther 10(5): 225.

[3] Adamopoulos, I. E, Sabokbar, A, et al. (2006). Synovial fluid macrophages are capable of osteoclast formation and resorption." J Pathol , 208(1), 35-43.

[4] Aguirre, J. I, Plotkin, L. I, et al. (2006). Osteocyte apoptosis is induced by weightlessness in mice and precedes osteoclast recruitment and bone loss." J Bone Miner Res , 21(4), 605-615.

[5] Aigner, T, Rose, J, et al. (2004). Aging theories of primary osteoarthritis: from epidemiology to molecular biology." Rejuvenation Res , 7(2), 134-145. 
[6] Arai, F, Miyamoto, T, et al. (1999). Commitment and differentiation of osteoclast precursor cells by the sequential expression of c-Fms and receptor activator of nuclear factor kappaB (RANK) receptors." J Exp Med , 190(12), 1741-1754.

[7] Boissier, M. C. (2011). Cell and cytokine imbalances in rheumatoid synovitis." Joint Bone Spine , 78(3), 230-234.

[8] Bonewald, L. F. (2007). Osteocytes as dynamic multifunctional cells." Ann N Y Acad Sci , 1116, 281-290.

[9] Burgess, T. L, Qian, Y, et al. (1999). The ligand for osteoprotegerin (OPGL) directly activates mature osteoclasts." J Cell Biol , 145(3), 527-538.

[10] Carvalheiro, H, \& Da, J. A. Silva, et al. ((2012). Potential roles for CD8(+) T cells in rheumatoid arthritis." Autoimmun Rev.

[11] Chang, M. K, Raggatt, L. J, et al. (2008). Osteal tissue macrophages are intercalated throughout human and mouse bone lining tissues and regulate osteoblast function in vitro and in vivo." J Immunol , 181(2), 1232-1244.

[12] Chen, F. H, \& Tuan, R. S. (2008). Mesenchymal stem cells in arthritic diseases." Arthritis Res Ther 10(5): 223.

[13] Cocucci, E, Racchetti, G, et al. (2009). Shedding microvesicles: artefacts no more." Trends Cell Biol , 19(2), 43-51.

[14] Davies, J, Warwick, J, et al. (1989). The osteoclast functional antigen, implicated in the regulation of bone resorption, is biochemically related to the vitronectin receptor." J Cell Biol 109(4 Pt 1): 1817-1826.

[15] De Broe, M. E, Wieme, R. J, et al. (1977). Spontaneous shedding of plasma membrane fragments by human cells in vivo and in vitro." Clin Chim Acta , 81(3), 237-245.

[16] De Smet, E, Jaecques, S. V, et al. (2007). Effect of constant strain rate, composed of varying amplitude and frequency, of early loading on peri-implant bone (re)modelling." J Clin Periodontol , 34(7), 618-624.

[17] Drake, F. H, Dodds, R. A, et al. (1996). Cathepsin K, but not cathepsins B, L, or S, is abundantly expressed in human osteoclasts." J Biol Chem , 271(21), 12511-12516.

[18] Everts, V, Delaisse, J. M, et al. (2002). The bone lining cell: its role in cleaning Howship's lacunae and initiating bone formation." J Bone Miner Res , 17(1), 77-90.

[19] Fermor, B, \& Skerry, T. M. (1995). PTH/PTHrP receptor expression on osteoblasts and osteocytes but not resorbing bone surfaces in growing rats." J Bone Miner Res, 10(12), 1935-1943.

[20] Franceschi, R. T, Xiao, G, et al. (2003). Multiple signaling pathways converge on the Cbfa1/Runx2 transcription factor to regulate osteoblast differentiation." Connect Tissue Res 44 Suppl , 1, 109-116. 
[21] Fritz, V, Brondello, J. M, et al. (2011). Bone-metastatic prostate carcinoma favors mesenchymal stem cell differentiation toward osteoblasts and reduces their osteoclastogenic potential." J Cell Biochem , 112(11), 3234-3245.

[22] Fujikawa, Y, Sabokbar, A, et al. (1996). Human osteoclast formation and bone resorption by monocytes and synovial macrophages in rheumatoid arthritis." Ann Rheum Dis , 55(11), 816-822.

[23] Fuller, K, Owens, J. M, et al. (1993). Macrophage colony-stimulating factor stimulates survival and chemotactic behavior in isolated osteoclasts." J Exp Med, 178(5), 1733-1744.

[24] Gao, Y, Ganss, B. W, et al. (2005). The RING finger protein RNF11 is expressed in bone cells during osteogenesis and is regulated by Ets1." Exp Cell Res, 304(1), 127-135.

[25] Gillespie, M. T. (2007). Impact of cytokines and T lymphocytes upon osteoclast differentiation and function." Arthritis Res Ther 9(2): 103.

[26] Goldring, S. R. (2012). Alterations in periarticular bone and cross talk between subchondral bone and articular cartilage in osteoarthritis." Ther Adv Musculoskelet Dis , 4(4), 249-258.

[27] Gordeladze, J, Djouad, F, Richard-duroux, I, Bony, C, Yssel, H, Nuber, U, Courties, G, Presumay, J, Noël, D, Brondello, J-M, Apparailly, F, Lecellier, C, \& Jorgensen, C. (2009). Strategy to identify a MicroRNA Signature of Chondrocytes as a Marker forDifferentiation from Mesenchymal Stem Cells." PNAS, in press.

[28] Gordeladze, J, Reseland, J. E, Karlsen, T. A, Jakobsen, R. B, Engebretsen, L, Lyngstadaas, S. P, Jorgensen, C, Yssel, H, \& Binchmann, J. E. (2011). Bone and Cartilage from Stem Cells: Growth Optimalization and Stabilization of Cell Phenotypes. In Regenerative Medicine and Tissue Enngineering ": , 23-56.

[29] Gordeladze, J. O, Djouad, F, et al. (2009). Concerted stimuli regulating osteo-chondral differentiation from stem cells: phenotype acquisition regulated by microRNAs." Acta pharmacologica Sinica, 30(10), 1369-1384.

[30] Gordeladze, J. O, Noel, D, et al. (2008). Transient down-regulation of cbfa1/Runx2 by RNA interference in murine C3H10T1/2 mesenchymal stromal cells delays in vitro and in vivo osteogenesis, but does not overtly affect chondrogenesis." Exp Cell Res , 314(7), 1495-1506.

[31] Gordeladze, J. O, Reseland, J. E, et al. (2009). From stem cells to bone: phenotype acquisition, stabilization, and tissue engineering in animal models." ILAR J , 51(1), 42-61.

[32] Harmey, D, Hessle, L, et al. (2004). Concerted regulation of inorganic pyrophosphate and osteopontin by akp2, enpp1, and ank: an integrated model of the pathogenesis of mineralization disorders." Am J Pathol , 164(4), 1199-1209. 
[33] Hassan, M. Q, Gordon, J. A, et al. (2010). A network connecting Runx2, SATB2, and the miR-23a 27a 24-2 cluster regulates the osteoblast differentiation program." Proc Natl Acad Sci U S A , 107(46), 19879-19884.

[34] Hattersley, G, \& Chambers, T. J. (1989). Calcitonin receptors as markers for osteoclastic differentiation: correlation between generation of bone-resorptive cells and cells that express calcitonin receptors in mouse bone marrow cultures." Endocrinology, 125(3), 1606-1612.

[35] Hayashi, D, Xu, L, et al. (2012). Detection of osteophytes and subchondral cysts in the knee with use of tomosynthesis." Radiology , 263(1), 206-215.

[36] Heijnen, H. F, Schiel, A. E, et al. (1999). Activated platelets release two types of membrane vesicles: microvesicles by surface shedding and exosomes derived from exocytosis of multivesicular bodies and alpha-granules." Blood , 94(11), 3791-3799.

[37] Heinemann, D. E, Siggelkow, H, et al. (2000). Alkaline phosphatase expression during monocyte differentiation. Overlapping markers as a link between monocytic cells, dendritic cells, osteoclasts and osteoblasts." Immunobiology , 202(1), 68-81.

[38] Heining, E, Bhushan, R, et al. (2011). Spatial segregation of BMP/Smad signaling affects osteoblast differentiation in C2C12 cells." PLoS One 6(10): e25163.

[39] Heino, T. J, Hentunen, T. A, et al. (2002). Osteocytes inhibit osteoclastic bone resorption through transforming growth factor-beta: enhancement by estrogen." J Cell Biochem , 85(1), 185-197.

[40] Hirota, K, Hashimoto, M, et al. (2007). T cell self-reactivity forms a cytokine milieu for spontaneous development of IL-17+ Th cells that cause autoimmune arthritis." J Exp Med , 204(1), 41-47.

[41] Hofbauer, L. C, Khosla, S, et al. (2000). The roles of osteoprotegerin and osteoprotegerin ligand in the paracrine regulation of bone resorption." J Bone Miner Res , 15(1), $2-12$.

[42] Holtrop, M. E, \& King, G. J. (1977). The ultrastructure of the osteoclast and its functional implications." Clin Orthop Relat Res(123): 177-196.

[43] Horton, M. A, Taylor, M. L, et al. (1991). Arg-Gly-Asp (RGD) peptides and the antivitronectin receptor antibody $23 \mathrm{C} 6$ inhibit dentine resorption and cell spreading by osteoclasts." Exp Cell Res , 195(2), 368-375.

[44] Hu, R, Sharma, S. M, et al. (2007). Eos, MITF, and PU.1 recruit corepressors to osteoclast-specific genes in committed myeloid progenitors." Mol Cell Biol , 27(11), 4018-4027.

[45] Hunter, M. P, Ismail, N, et al. (2008). Detection of microRNA expression in human peripheral blood microvesicles." PLoS One 3(11): e3694. 
[46] Insogna, K. L, Sahni, M, et al. (1997). Colony-stimulating factor-1 induces cytoskeletal reorganization and c-src-dependent tyrosine phosphorylation of selected cellular proteins in rodent osteoclasts." J Clin Invest , 100(10), 2476-2485.

[47] Iorio, M. V, Casalini, P, et al. (2011). Breast cancer and microRNAs: therapeutic impact." Breast 20 Suppl 3: S, 63-70.

[48] Iwamoto, T, Okamoto, H, et al. (2008). Molecular aspects of rheumatoid arthritis: chemokines in the joints of patients." FEBS J , 275(18), 4448-4455.

[49] Juppner, H, Abou-samra, A. B, et al. (1991). A G protein-linked receptor for parathyroid hormone and parathyroid hormone-related peptide." Science, 254(5034), 1024-1026.

[50] Karsenty, G. (2008). Transcriptional control of skeletogenesis." Annu Rev Genomics Hum Genet , 9, 183-196.

[51] Kastrinaki, M. C, \& Papadaki, H. A. (2009). Mesenchymal stromal cells in rheumatoid arthritis: biological properties and clinical applications." Curr Stem Cell Res Ther , $4(1), 61-69$.

[52] Kirkham, B. W, Lassere, M. N, et al. (2006). Synovial membrane cytokine expression is predictive of joint damage progression in rheumatoid arthritis: a two-year prospective study (the DAMAGE study cohort)." Arthritis Rheum , 54(4), 1122-1131.

[53] Kochi, Y. (2010). Genetic background of tolerance breakdown in rheumatoid arthritis." Nihon Rinsho Meneki Gakkai Kaishi , 33(2), 48-56.

[54] Koenders, M. I, Lubberts, E, et al. (2005). Blocking of interleukin-17 during reactivation of experimental arthritis prevents joint inflammation and bone erosion by decreasing RANKL and interleukin-1." Am J Pathol , 167(1), 141-149.

[55] Komatsu, T, Mizusaki, H, et al. (2004). Small ubiquitin-like modifier 1 (SUMO-1) modification of the synergy control motif of Ad4 binding protein/steroidogenic factor 1 (Ad4BP/SF-1) regulates synergistic transcription between Ad4BP/SF-1 and Sox9." Mol Endocrinol , 18(10), 2451-2462.

[56] Kraal, G, Rep, M, et al. (1987). Macrophages in T and B cell compartments and other tissue macrophages recognized by monoclonal antibody MOMA-2. An immunohistochemical study." Scand J Immunol , 26(6), 653-661.

[57] Kular, J, Tickner, J, et al. (2012). An overview of the regulation of bone remodelling at the cellular level." Clin Biochem , 45(12), 863-873.

[58] Lacey, D. L, Timms, E, et al. (1998). Osteoprotegerin ligand is a cytokine that regulates osteoclast differentiation and activation." Cell , 93(2), 165-176.

[59] Langrish, C. L, Chen, Y, et al. (2005). IL-23 drives a pathogenic T cell population that induces autoimmune inflammation." J Exp Med , 201(2), 233-240. 
[60] Larsen, M, Arnaud, L, et al. (2011). Multiparameter grouping delineates heterogeneous populations of human IL-17 and/or IL-22 T-cell producers that share antigen specificities with other T-cell subsets." Eur J Immunol , 41(9), 2596-2605.

[61] Le BechecA., E. Portales-Casamar, et al. ((2011). MIR@NT@N: a framework integrating transcription factors, microRNAs and their targets to identify sub-network motifs in a meta-regulation network model." BMC Bioinformatics 12: 67.

[62] Legendre, F, Dudhia, J, et al. (2003). JAK/STAT but not ERK1/ERK2 pathway mediates interleukin (IL)-6/soluble IL-6R down-regulation of Type II collagen, aggrecan core, and link protein transcription in articular chondrocytes. Association with a down-regulation of SOX9 expression." J Biol Chem , 278(5), 2903-2912.

[63] Li, X, Qin, L, et al. (2007). Parathyroid hormone stimulates osteoblastic expression of MCP-1 to recruit and increase the fusion of pre/osteoclasts." J Biol Chem , 282(45), 33098-33106.

[64] Li, X, Zhang, Y, et al. (2005). Sclerostin binds to LRP5/6 and antagonizes canonical Wnt signaling." J Biol Chem , 280(20), 19883-19887.

[65] Li, Y, Toraldo, G, et al. (2007). B cells and T cells are critical for the preservation of bone homeostasis and attainment of peak bone mass in vivo." Blood, 109(9), 3839-3848.

[66] Li, Z, Hassan, M. Q, et al. (2009). Biological functions of miR-29b contribute to positive regulation of osteoblast differentiation." J Biol Chem.

[67] Li, Z, Hassan, M. Q, et al. (2008). A microRNA signature for a BMP2-induced osteoblast lineage commitment program." Proc Natl Acad Sci U S A , 105(37), 13906-13911.

[68] Lian, J. B, Javed, A, et al. (2004). Regulatory controls for osteoblast growth and differentiation: role of Runx/Cbfa/AML factors." Crit Rev Eukaryot Gene Expr 14(1-2): 1-41.

[69] Lin, G. L, \& Hankenson, K. D. (2011). Integration of BMP, Wnt, and notch signaling pathways in osteoblast differentiation." J Cell Biochem , 112(12), 3491-3501.

[70] Lories, R. (2011). The balance of tissue repair and remodeling in chronic arthritis." Nat Rev Rheumatol , 7(12), 700-707.

[71] Lu, X, Beck, G. R, et al. (2011). Identification of the homeobox protein Prx1 (MHox, Prrx-1) as a regulator of osterix expression and mediator of tumor necrosis factor alpha action in osteoblast differentiation." J Bone Miner Res , 26(1), 209-219.

[72] Lubberts, E, Koenders, M. I, et al. (2004). Treatment with a neutralizing anti-murine interleukin-17 antibody after the onset of collagen-induced arthritis reduces joint inflammation, cartilage destruction, and bone erosion." Arthritis Rheum , 50(2), 650-659.

[73] Luchin, A, Purdom, G, et al. (2000). The microphthalmia transcription factor regulates expression of the tartrate-resistant acid phosphatase gene during terminal differentiation of osteoclasts." J Bone Miner Res , 15(3), 451-460. 
[74] Luzi, E, Marini, F, et al. (2012). The regulatory network menin-microRNA 26a as a possible target for RNA-based therapy of bone diseases." Nucleic Acid Ther , 22(2), 103-108.

[75] Ma, Y. L, Cain, R. L, et al. (2001). Catabolic effects of continuous human PTH (1--38) in vivo is associated with sustained stimulation of RANKL and inhibition of osteoprotegerin and gene-associated bone formation." Endocrinology , 142(9), 4047-4054.

[76] Mackie, E. J, Ahmed, Y. A, et al. (2008). Endochondral ossification: how cartilage is converted into bone in the developing skeleton." Int J Biochem Cell Biol , 40(1), 46-62.

[77] Majka, M, Janowska-wieczorek, A, et al. (2001). Numerous growth factors, cytokines, and chemokines are secreted by human CD34(+) cells, myeloblasts, erythroblasts, and megakaryoblasts and regulate normal hematopoiesis in an autocrine/paracrine manner." Blood , 97(10), 3075-3085.

[78] Manel, N, Unutmaz, D, et al. (2008). The differentiation of human T(H)-17 cells requires transforming growth factor-beta and induction of the nuclear receptor RORgammat." Nat Immunol , 9(6), 641-649.

[79] Manolagas, S. C, \& Almeida, M. (2007). Gone with the Wnts: beta-catenin, T-cell factor, forkhead box $\mathrm{O}$, and oxidative stress in age-dependent diseases of bone, lipid, and glucose metabolism." Mol Endocrinol , 21(11), 2605-2614.

[80] Marie, P. J. (2008). Transcription factors controlling osteoblastogenesis." Arch Biochem Biophys , 473(2), 98-105.

[81] Martin, T. J, \& Sims, N. A. (2005). Osteoclast-derived activity in the coupling of bone formation to resorption." Trends Mol Med , 11(2), 76-81.

[82] Matsumoto, M, Kogawa, M, et al. (2004). Essential role of mitogen-activated protein kinase in cathepsin $\mathrm{K}$ gene expression during osteoclastogenesis through association of NFATc1 and PU.1." J Biol Chem 279(44): 45969-45979., 38.

[83] Mazzola, L, Bemporad, E, et al. (2011). Surface analysis and osteoblasts response of a titanium oxi-carbide film deposited on titanium by ion plating plasma assisted (IPPA)." J Nanosci Nanotechnol , 11(10), 8754-8762.

[84] Mchugh, K. P, Hodivala-dilke, K, et al. (2000). Mice lacking beta3 integrins are osteosclerotic because of dysfunctional osteoclasts." J Clin Invest , 105(4), 433-440.

[85] Mellis, D. J, Itzstein, C, et al. (2011). The skeleton: a multi-functional complex organ: the role of key signalling pathways in osteoclast differentiation and in bone resorption." J Endocrinol , 211(2), 131-143.

[86] Minkin, C. (1982). Bone acid phosphatase: tartrate-resistant acid phosphatase as a marker of osteoclast function." Calcif Tissue Int , 34(3), 285-290. 
[87] Moretti, F, Thermann, R, et al. (2010). Mechanism of translational regulation by miR-2 from sites in the $5^{\prime}$ untranslated region or the open reading frame." $R N A$, 16(12), 2493-2502.

[88] Motyl, K. J, \& Rosen, C. J. (2012). Understanding leptin-dependent regulation of skeletal homeostasis." Biochimie.

[89] Murshed, M, Harmey, D, et al. (2005). Unique coexpression in osteoblasts of broadly expressed genes accounts for the spatial restriction of ECM mineralization to bone." Genes Dev , 19(9), 1093-1104.

[90] Muys, J. J, Alkaisi, M. M, et al. (2006). Cellular transfer and AFM imaging of cancer cells using Bioimprint." J Nanobiotechnology 4: 1.

[91] Nakashima, T, Hayashi, M, et al. (2012). New insights into osteoclastogenic signaling mechanisms." Trends Endocrinol Metab.

[92] Nakashima, T, \& Takayanagi, H. (2009). Osteoimmunology: crosstalk between the immune and bone systems." J Clin Immunol , 29(5), 555-567.

[93] Neve, A, Corrado, A, et al. (2011). Osteoblast physiology in normal and pathological conditions." Cell Tissue Res , 343(2), 289-302.

[94] Newby, A. C. (2008). Metalloproteinase expression in monocytes and macrophages and its relationship to atherosclerotic plaque instability." Arterioscler Thromb Vasc Biol , 28(12), 2108-2114.

[95] Oh, H. J, Kido, T, et al. (2007). PIAS1 interacts with and represses SOX9 transactivation activity." Mol Reprod Dev , 74(11), 1446-1455.

[96] Palumbo, C. ultrastructural study of osteoid-osteocytes in the tibia of chick embryos." Cell Tissue Res , 246(1), 125-131.

[97] Parsonage, G, Filer, A, et al. (2008). Prolonged, granulocyte-macrophage colony-stimulating factor-dependent, neutrophil survival following rheumatoid synovial fibroblast activation by IL-17 and TNFalpha." Arthritis Res Ther 10(2): R47.

[98] Partridge, N. C, Jeffrey, J. J, et al. (1987). Hormonal regulation of the production of collagenase and a collagenase inhibitor activity by rat osteogenic sarcoma cells." Endocrinology , 120(5), 1956-1962.

[99] Pederson, L, Ruan, M, et al. (2008). Regulation of bone formation by osteoclasts involves Wnt/BMP signaling and the chemokine sphingosine-1-phosphate." Proc Natl Acad Sci U S A , 105(52), 20764-20769.

[100] Pene, J, Chevalier, S, et al. (2008). Chronically inflamed human tissues are infiltrated by highly differentiated Th17 lymphocytes." J Immunol , 180(11), 7423-7430. 
[101] Quinn, J. M, Elliott, J, et al. (1998). A combination of osteoclast differentiation factor and macrophage-colony stimulating factor is sufficient for both human and mouse osteoclast formation in vitro." Endocrinology , 139(10), 4424-4427.

[102] Raggatt, L. J, \& Partridge, N. C. (2010). Cellular and molecular mechanisms of bone remodeling." J Biol Chem , 285(33), 25103-25108.

[103] Ratajczak, J, Wysoczynski, M, et al. (2006). Membrane-derived microvesicles: important and underappreciated mediators of cell-to-cell communication." Leukemia , 20(9), 1487-1495.

[104] Robling, A. G, Niziolek, P. J, et al. (2008). Mechanical stimulation of bone in vivo reduces osteocyte expression of Sost/sclerostin." J Biol Chem , 283(9), 5866-5875.

[105] Rozmyslowicz, T, Majka, M, et al. (2003). Platelet- and megakaryocyte-derived microparticles transfer CXCR4 receptor to CXCR4-null cells and make them susceptible to infection by X4-HIV." AIDS , 17(1), 33-42.

[106] Rustom, A, Saffrich, R, et al. (2004). Nanotubular highways for intercellular organelle transport." Science , 303(5660), 1007-1010.

[107] Saftig, P, Hunziker, E, et al. (1998). Impaired osteoclastic bone resorption leads to osteopetrosis in cathepsin-K-deficient mice." Proc Natl Acad Sci U S A, 95(23), 13453-13458.

[108] Schoof, C. R, Botelho, E. L, et al. (2012). MicroRNAs in cancer treatment and prognosis." Am J Cancer Res , 2(4), 414-433.

[109] Shegarfi, H, Naddafi, F, et al. (2012). Natural killer cells and their role in rheumatoid arthritis: friend or foe?" ScientificWorldJournal 2012: 491974.

[110] Sherer, N. M, \& Mothes, W. (2008). Cytonemes and tunneling nanotubules in cell-cell communication and viral pathogenesis." Trends Cell Biol , 18(9), 414-420.

[111] Siekevitz, P. (1972). Biological membranes: the dynamics of their organization." Annu Rev Physiol , 34, 117-140.

[112] Simonet, W. S, Lacey, D. L, et al. (1997). Osteoprotegerin: a novel secreted protein involved in the regulation of bone density." Cell , 89(2), 309-319.

[113] Sims, N. A, \& Walsh, N. C. (2012). Intercellular cross-talk among bone cells: new factors and pathways." Curr Osteoporos Rep , 10(2), 109-117.

[114] Suzuki, T, Osumi, N, et al. (2010). Stabilization of ATF4 protein is required for the regulation of epithelial-mesenchymal transition of the avian neural crest." Dev Biol , 344(2), 658-668.

[115] Swarthout, J. T, Alonzo, R. C. D, et al. (2002). Parathyroid hormone-dependent signaling pathways regulating genes in bone cells." Gene 282(1-2): 1-17. 
[116] Takahashi, F, Takahashi, K, et al. (2004). Osteopontin is strongly expressed by alveolar macrophages in the lungs of acute respiratory distress syndrome." Lung , 182(3), 173-185.

[117] Takarada, T, \& Yoneda, Y. (2008). Pharmacological topics of bone metabolism: glutamate as a signal mediator in bone." J Pharmacol Sci, 106(4), 536-541.

[118] Takayanagi, H, Iizuka, H, et al. (2000). Involvement of receptor activator of nuclear factor kappaB ligand/osteoclast differentiation factor in osteoclastogenesis from synoviocytes in rheumatoid arthritis." Arthritis Rheum , 43(2), 259-269.

[119] Tan, Y. K, \& Conaghan, P. G. (2011). Imaging in rheumatoid arthritis." Best Pract Res Clin Rheumatol , 25(4), 569-584.

[120] Tang, Y, Wu, X, et al. (2009). TGF-beta1-induced migration of bone mesenchymal stem cells couples bone resorption with formation." Nat Med , 15(7), 757-765.

[121] Tani, A, Tada, Y, et al. (2012). Regeneration of tracheal epithelium using a collagen vitrigel-sponge scaffold containing basic fibroblast growth factor." Ann Otol Rhinol Laryngol , 121(4), 261-268.

[122] Teitelbaum, S. L. (2000). Bone resorption by osteoclasts." Science , 289(5484), 1504-1508.

[123] Teitelbaum, S. L, \& Ross, F. P. (2003). Genetic regulation of osteoclast development and function." Nat Rev Genet , 4(8), 638-649.

[124] Thompson, W. R, Rubin, C. T, et al. (2012). Mechanical regulation of signaling pathways in bone." Gene , 503(2), 179-193.

[125] Tondravi, M. M, Mckercher, S. R, et al. (1997). Osteopetrosis in mice lacking haematopoietic transcription factor PU.1." Nature , 386(6620), 81-84.

[126] Tran, P, Vignery, A, et al. (1982). An electron-microscopic study of the bone-remodeling sequence in the rat." Cell Tissue Res , 225(2), 283-292.

[127] Van Bezooijen, R. L, Roelen, B. A, et al. (2004). Sclerostin is an osteocyte-expressed negative regulator of bone formation, but not a classical BMP antagonist." J Exp Med, 199(6), 805-814.

[128] Van Der Kraan, P. M. and W. B. van den Berg ((2012). Chondrocyte hypertrophy and osteoarthritis: role in initiation and progression of cartilage degeneration?" Osteoarthritis Cartilage , 20(3), 223-232.

[129] Verborgt, O, Tatton, N. A, et al. (2002). Spatial distribution of Bax and Bcl-2 in osteocytes after bone fatigue: complementary roles in bone remodeling regulation?" J Bone Miner Res , 17(5), 907-914. 
[130] Volpe, E, Servant, N, et al. (2008). A critical function for transforming growth factorbeta, interleukin 23 and proinflammatory cytokines in driving and modulating human T(H)-17 responses." Nat Immunol , 9(6), 650-657.

[131] Weitzmann, M. N, \& Pacifici, R. unexpected players in the bone loss induced by estrogen deficiency and in basal bone homeostasis." Ann N Y Acad Sci, 1116, 360-375.

[132] Wilson, N. J, Boniface, K, et al. (2007). Development, cytokine profile and function of human interleukin 17-producing helper T cells." Nat Immunol , 8(9), 950-957.

[133] Woo, K. M, Kim, H. M, et al. (2002). Macrophage colony-stimulating factor promotes the survival of osteoclast precursors by up-regulating Bcl-X(L)." Exp Mol Med, 34(5), 340-346.

[134] Xiong, J, Onal, M, et al. (2011). Matrix-embedded cells control osteoclast formation." Nat Med , 17(10), 1235-1241.

[135] Yamada, H, Nakashima, Y, et al. (2008). Th1 but not Th17 cells predominate in the joints of patients with rheumatoid arthritis." Ann Rheum Dis , 67(9), 1299-1304.

[136] Yamashiro, T, Fukunaga, T, et al. (2001). Gene and protein expression of brain-derived neurotrophic factor and TrkB in bone and cartilage." Bone , 28(4), 404-409.

[137] Yang, C. M, Chien, C. S, et al. (2004). Mechanical strain induces collagenase-3 (MMP-13) expression in MC3T3-E1 osteoblastic cells." J Biol Chem , 279(21), 22158-22165.

[138] Yang, L, Anderson, D. E, et al. (2008). IL-21 and TGF-beta are required for differentiation of human T(H)17 cells." Nature , 454(7202), 350-352.

[139] Yanni, G, Whelan, A, et al. (1994). Synovial tissue macrophages and joint erosion in rheumatoid arthritis." Ann Rheum Dis , 53(1), 39-44.

[140] Zhang, Y, Xie, R. L, et al. (2011). A program of microRNAs controls osteogenic lineage progression by targeting transcription factor Runx2." Proc Natl Acad Sci U S A, 108(24), 9863-9868.

[141] Zhao, C, Irie, N, et al. (2006). Bidirectional ephrinB2-EphB4 signaling controls bone homeostasis." Cell Metab ,4(2), 111-121.

[142] Aarvak, T, Chabaud, M, et al. (1999). IL-17 is produced by some proinflammatory Th1/Th0 cells but not by Th2 cells." J Immunol , 162(3), 1246-1251. 
